\title{
Stability Analysis for HIFiRE Experiments
}

\author{
Fei Li, ${ }^{*}$ Meelan Choudhari, ${ }^{* *}$ Chau-Lyan Chang ${ }^{+}$, Jeffery White ${ }^{++}$ \\ NASA Langley Research Center, Hampton, VA 23681 \\ Roger Kimmel ${ }^{\#}$, David Adamczak ${ }^{\&}$, Matthew Borg ${ }^{+++}$and Scott Stanfield, ${ }^{++++}$ \\ Air Force Research Laboratory, WPAFB, OH 45433 \\ Mark Smith"\# \\ NASA Dryden Research Center, Edwards, CA 93523
}

\begin{abstract}
The HIFiRE-1 flight experiment provided a valuable database pertaining to boundary layer transition over a 7-degree half-angle, circular cone model from supersonic to hypersonic Mach numbers, and a range of Reynolds numbers and angles of attack. This paper reports selected findings from the ongoing computational analysis of the measured in-flight transition behavior. Transition during the ascent phase at nearly zero degree angle of attack is dominated by second mode instabilities except in the vicinity of the cone meridian where a roughness element was placed midway along the length of the cone. The growth of first mode instabilities is found to be weak at all trajectory points analyzed from the ascent phase. For times less than approximately 18.5 seconds into the flight, the peak amplification ratio for second mode disturbances is sufficiently small because of the lower Mach numbers at earlier times, so that the transition behavior inferred from the measurements is attributed to an unknown physical mechanism, potentially related to step discontinuities in surface height near the locations of a change in the surface material. Based on the time histories of temperature and/or heat flux at transducer locations within the aft portion of the cone, the onset of transition correlated with a linear $\mathrm{N}$-factor, based on parabolized stability equations, of approximately 13.5. Due to the large angles of attack during the re-entry phase, crossflow instability may play a significant role in transition. Computations also indicate the presence of pronounced crossflow separation over a significant portion of the trajectory segment that is relevant to transition analysis. The transition behavior during this re-entry segment of HIFiRE-1 flight shares some common features with the predicted transition front along the elliptic cone shaped HIFiRE-5 flight article, which was designed to provide hypersonic transition data for a fully 3D geometric configuration. To compare and contrast the crossflow dominated transition over the HIFiRE-1 and HIFiRE-5 configurations, this paper also analyzes boundary layer instabilities over a subscale model of the HIFiRE-5 flight configuration that was tested in the Mach 6 quiet tunnel facility at Purdue University.
\end{abstract}

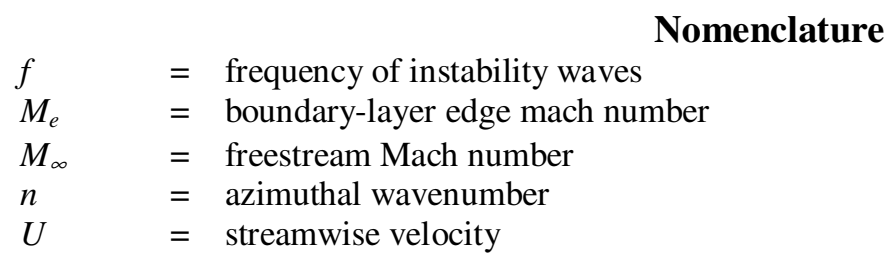

\footnotetext{
* Aerospace Technologist, Computational AeroSciences Branch, M.S. 128.

*** Aerospace Technologist, Computational AeroSciences Branch, M.S. 128, Associate Fellow, AIAA.

+ Aerospace Technologist, Computational AeroSciences Branch, M.S. 128, Senior Member, AIAA.

${ }^{++}$Aerospace Technologist, Computational AeroSciences Branch, M.S. 128, Senior Member, AIAA.

\# Senior Research Engineer, Air Vehicle Directorate, 2130 8th St., WPAFB, OH 45433, Associate Fellow, AIAA.

\& Senior Aerospace Engineer, Air Vehicle Directorate, 2130 8th St., WPAFB, OH 45433.

${ }^{+++}$Booz Allen Hamilton, Member, AIAA.

${ }^{++++}$Spectral Energies, Member, AIAA.

\#\# Aerospace Engineer, Aerodynamics and Propulsion Branch, M.S. 2228.
}

1

American Institute of Aeronautics and Astronautics 


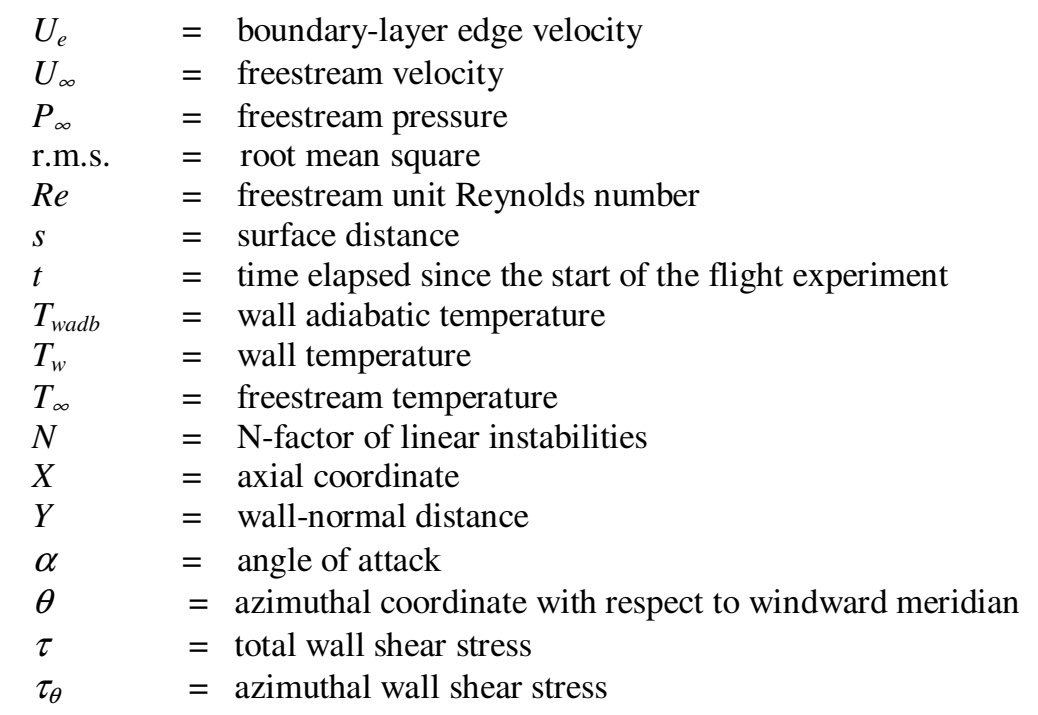

\section{Introduction}

The Hypersonic International Flight Research and Experimentation (HIFiRE) series of flight experiments by the U.S. Air Force Research Laboratory (AFRL) and Australian Defense Science and Technology is designed to demonstrate fundamental technologies critical to the next generation aerospace systems. The aim of the first of these experiments, HIFiRE-1, was to obtain in-flight transitional and turbulent boundary layer heating data on a 7-deg cone-cylinder-flare configuration. The follow-on HIFiRE-5 experiment is designed to provide transition data for an elliptic cone, i.e., fully 3D flow configuration. The present computational analysis is aimed at characterizing the laminar-turbulent transition over the surface of the HIFiRE-1 cone and comparing the predicted transition behavior with that inferred from the flight and/or wind tunnel measurements and direct numerical simulations. The primary technical objectives are to test and validate the state of the art transition prediction tools for flow configurations with multiple modes of instability, to establish transition correlation criteria against flight data for both simple (i.e., axisymmetric) and fully 3D flow configurations, and to examine the sensitivities of transition characteristics to uncertainties in flight conditions.

The design of the HIFiRE-1 flight experiment and the associated pre-flight effort are summarized in Refs. 1 and 2, and the analysis of the actual flight data is discussed in Refs. 3-5. The primary configuration for the transition measurement in the HIFiRE-1 flight experiment corresponds to a circular cone of 1.1 meters in length, with a cone half angle of 7 degrees and a small nose radius of $2.5 \mathrm{~mm}$. The first and last 45 seconds of the flight were endo-atmospheric (i.e., inside the atmosphere) and, hence, are potentially relevant to post-flight transition studies. In the ascending phase, the transition front is observed to move off the cone at $t=23$ seconds into the flight, leaving behind a laminar boundary layer. During the descending (or re-entry) phase, transition to turbulence first appears at $t=483.5$ seconds into the flight, based on the estimates of Refs. 4 and 5, and is last observed at $t=485$ seconds.

Reference 6 outlined computational results pertaining to transition analysis and correlations for a selected portion of the ascent phase of the HIFiRE-1 model trajectory corresponding to near-zero angle of attack. This paper presents a broader set of results including a larger portion of the ascent trajectory and a preliminary analysis of selected conditions from the descent phase of the flight trajectory. The descent phase exhibited significant deviations from the design trajectory, resulting in transition under angles of attack that were comparable to or even larger than the cone half-angle of 7 degrees. Thus, while flow conditions in the ascending phase of the HIFiRE-1 flight led to transition due to second-mode instability at sufficiently high values of the flight Mach number, both second mode and crossflow instabilities may be relevant to the transition process during the descending phase, in a way, similar to the transition mechanisms that are expected on the elliptic cone configuration from the HIFiRE-5 flight 
experiment $^{7,8}$. Selected results pertaining to both second mode and crossflow instability over the HIFiRE-1 configuration are presented in sections 2 and 3, respectively. Analogous results for a subscale wind tunnel model of the HIFiRE-5 flight configuration are presented in Section 4.

\section{HIFiRE-1 Transition Analysis during Ascent Phase}

The mean boundary layer flow over the cone surface was computed on various grids using a second order accurate algorithm as implemented in a finite-volume, structured grid, compressible Navier-Stokes flow solver VULCAN ${ }^{9}$. The VULCAN computations utilized the code's built-in capability to accomplish shock adaptations. Extensive grid convergence tests were performed to ensure that the mean flow solutions were sufficiently resolved for the purpose of stability analysis. The surface temperature distribution imposed during the mean flow computations was obtained by combining the results of thermal analysis based on axisymmetric, finite element calculations using AFRL's TOPAZ code ${ }^{2,3}$ and the experimental data based on thermocouple measurements.

The stability of the computed boundary layer flow was analyzed using the Langley Stability and Transition Analysis Code (LASTRAC) ${ }^{10}$. Most of the analysis was performed using parabolized stability equations (PSE), but the classical, quasi-parallel stability theory was used for the analysis of crossflow instabilities during the reentry phase. Sutherland's law is assumed to describe the viscosity variation for both the base flow and the unsteady perturbations associated with boundary layer instability waves. Stokes' law is assumed for bulk viscosity.

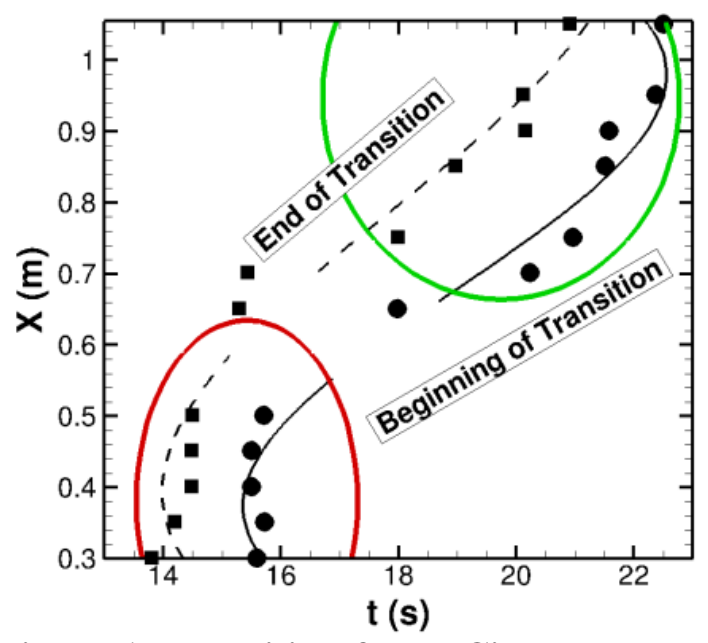

Figure 1. Transition front. Circular and square symbols represent, respectively, experimentally measured onset and ending of transition as given in Ref. 3. Lines represent curve fit through experimental data. Solid line is a curve fit through transition onset data and the dashed line is a curve fit through end-of-transition data. Red ellipse encloses region of rapid movement in transition front and green ellipse encloses region of slower transition front movement.
It should be emphasized at the outset that the transition in the ascending phase occurs via laminarization, i.e. initially turbulent boundary layer becomes laminar as the transition front moves downstream in response to the progressively smaller Reynolds number at higher altitude. The experimentally determined transition locations from Table 3 of Ref. 3 are plotted in Fig. 1 for a small time window in the ascending phase that corresponds to approximately 14 to 23 seconds into the flight. The times corresponding to both the boundary between laminar and transitional flow (i.e. "beginning of transition") and the boundary between transitional and fully turbulent flow (i.e., "end of transition") are plotted in the figure. The determination of transition is somewhat subjective, and depends on the criteria and type of sensor used for detection. In these results for ascent, transition end is defined as the time corresponding to a well-defined departure from the turbulent heat transfer, as measured by thermocouples. Transition onset is the time at which heat transfer appears to have fully relaxed to a laminar value.

During the window between 14 and 23 seconds, the transition onset front moves from $X=0.3 \mathrm{~m}$ over the first half of cone length to $1.05 \mathrm{~m}$ near the end of the cone. The circular and square symbols represent, respectively, the onset and the ending of transition. The lines are curve fits through these data points. The solid line is a curve fit for the onset of transition and the dashed line is a curve fit for the end of transition. 
Two distinct regions in Figure 1 can be identified: (1) a region of rapid transition front movement (enclosed by the red ellipse), in which transition happens almost simultaneously upstream of $X=0.5 \mathrm{~m}$, and (2) a region of relatively gradual transition front movement (enclosed by the green ellipse). According to Ref. 3, the rapid transition behavior may be attributed to a boundary-layer tripping effect due to the existence of two locations of material discontinuities at approximately $X=0.1$ and $0.2 \mathrm{~m}$, respectively. The latter region enclosed by the green ellipse in Figure 1 leaves a window of approximately 5 seconds in which second-mode $\mathrm{N}$-factor correlations with the experiment can be carried out.

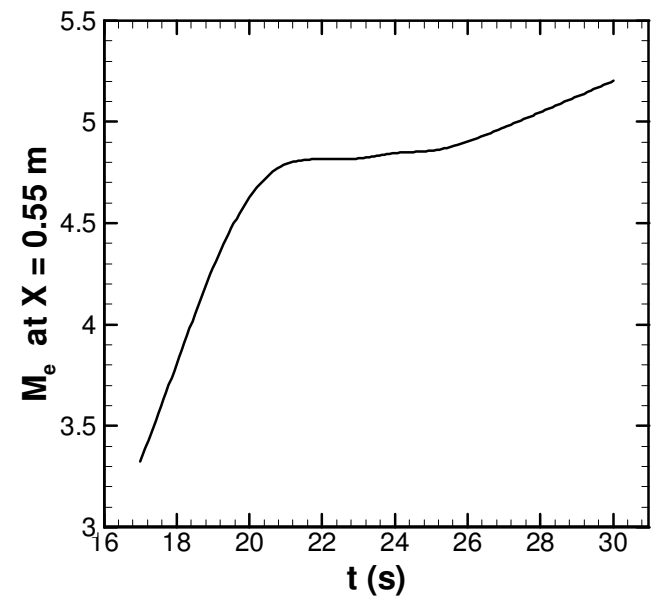

(a) Boundary-layer edge Mach number

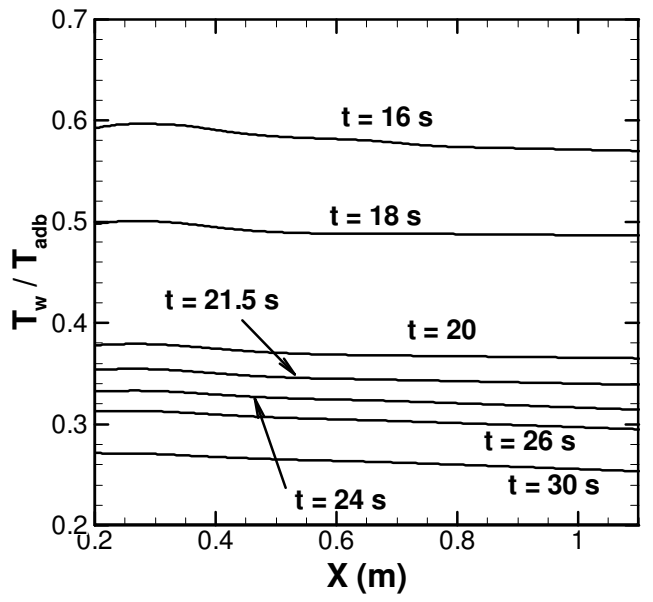

(b) Temperature ratio

Figure 2. Boundary-layer edge Mach number, $M_{e}$, at $X=0.55 \mathrm{~m}$ and ratio of wall temperature, $T_{w}$, to wall adiabatic temperature, $T_{a d b}$, during selected time interval from the ascent phase.

Mean flow computations are carried out for different instances of flight from 16 to 23 seconds in intervals of 1 second (and 0.5 seconds, occasionally). The boundary-layer edge Mach number at $X=0.55 \mathrm{~m}$ (midcone) is shown in Fig. 2(a) as a function of time and the ratio of wall temperature to wall adiabatic temperature along the cone are shown in Fig. 2(b) for selected cases. Similar to many hypersonic flight configurations, the surface temperatures downstream of the nose are considerably smaller than the local temperatures corresponding to an adiabatic thermal boundary condition. Thus, no significant first mode instability is expected during the selected window from the ascent phase and this was confirmed by the calculations. The linear instability phase is, therefore, dominated by second mode disturbances. The robustness of findings with respect to uncertainty in surface temperatures was directly confirmed via computations for different temperature distributions.

Based on these mean flow computations, $\mathrm{N}$-factors for each chosen instant of flight in this time range are computed. The maximum $\mathrm{N}$-factors and the corresponding frequencies are shown in Fig. 3. It can be seen that the mean flow at $t=20$ seconds gives rise to the largest maximum $\mathrm{N}$-factor of $N=21.2$ at the end of the cone among all instants for which computations were performed. By $t=27$ seconds, the maximum Nfactor drops to 7.6, indicating a progressively less unstable boundary layer. At the other end of the time window, $t=17$ seconds, the maximum $\mathrm{N}$-factor is only 3.5, and yet the experiment shows that there exists large regions of turbulent flow over the cone. This may also be attributed to the tripping effect discussed earlier. 


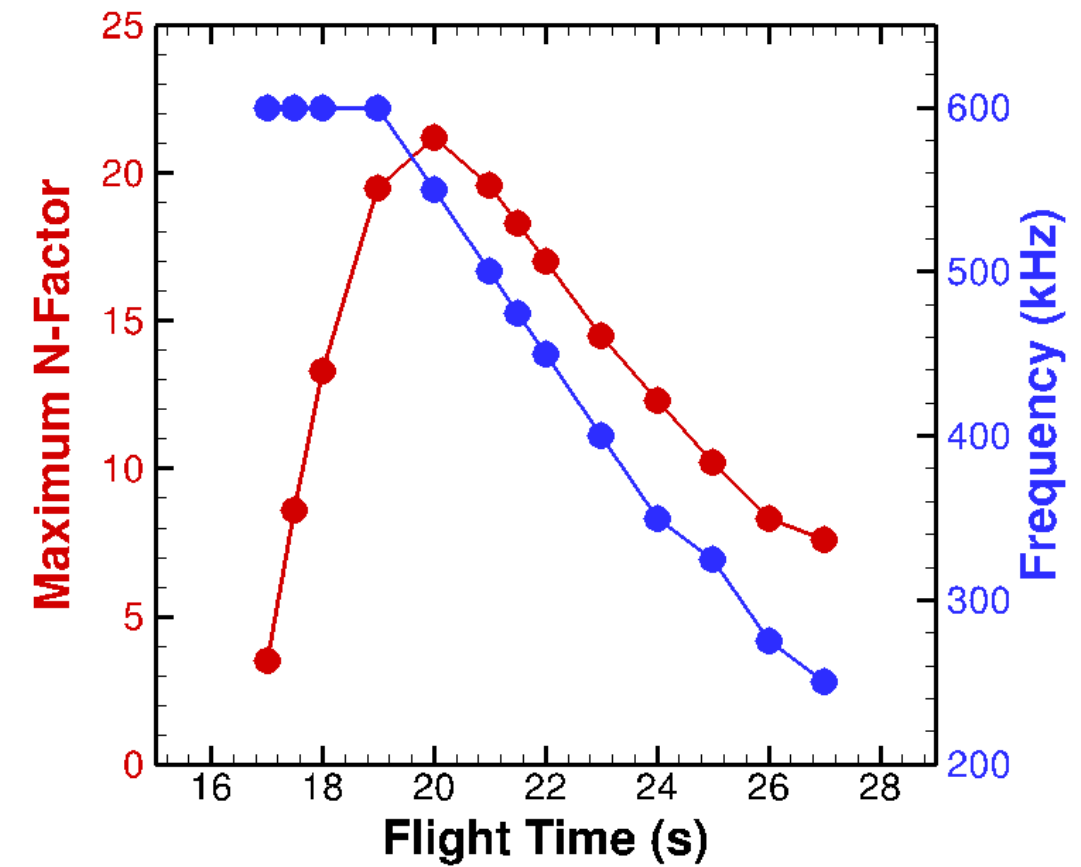

Figure 3. Temporal variation of maximum $\mathrm{N}$-factor over the length of the cone (red line and symbols) and the corresponding second mode frequency (blue line and symbols) during the trajectory segment of interest during ascent.

Based on the N-factor computations (Fig. 4) and experimental data (the portion of the fitted curve for the onset of transition that falls within the green ellipse in Figure 1) between 18 and 23 seconds into the flight, the $\mathrm{N}$-factor value correlating with the onset of transition was found to be approximately 13.5. To avoid confusion, it is stressed that this is the transition onset $\mathrm{N}$-factor, not the maximum $\mathrm{N}$-factor discussed in the previous paragraph. Quantifiable contributions to the uncertainty in $\mathrm{N}$-factor correlation were also determined. The factors influencing the accuracy of the $\mathrm{N}$-factor correlation include: uncertainties in the surface temperature distribution which must be specified as a boundary condition during mean flow computations; uncertainties in the actual angle of attack during the flight; and uncertainties about the freestream conditions of the flight at each instant of the trajectory. The effect of these uncertainties on the $\mathrm{N}$-factor correlation was found to be small. The uncertainty due to some other factors was difficult to quantify and, hence, was not addressed. One such factor corresponds to the uncertainty in the data reduction process involving the time histories of surface temperature and/or heat flux measured by the thermocouples and heat flux gauges, respectively. It pertains to the noise in the raw measurements and its impact on the estimates of the times for the onset and the end of transition, respectively. Furthermore, determining the times for the onset and the end of transition based on the inferred time histories at a given transducer location also involved some subjectivity as noted in Refs. 3 and 6.

The effect of changes in the surface geometry due to steps near material discontinuities was addressed in Ref. 6, wherein the effect of backward facing steps on second mode amplification was computed. It was found that, because the anticipated step locations near the material discontinuities on the model $(X=$ $0.113 \mathrm{~m}$ and $0.213 \mathrm{~m}$ ) were sufficiently farther upstream of the range of amplification for the relevant second mode disturbances, the effect of steps on second mode amplification during the trajectory window of interest was deemed to be secondary. Of course, this does not rule out other mechanisms by which the step excrescences could have influenced transition (e.g., increased receptivity, seeding of streamwise 
vorticity via azimuthal variations in step height, etc.). Investigation of these mechanisms was deferred to a future study.

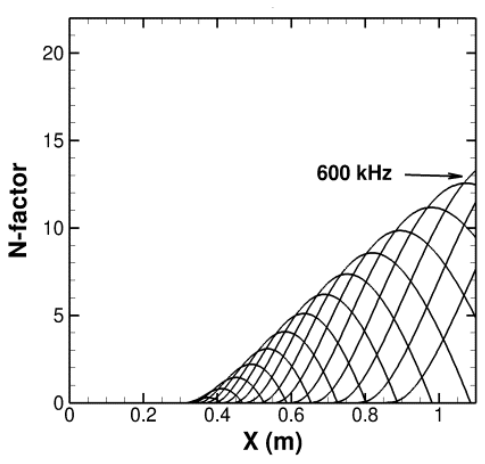

(a) $t=18 \mathrm{~s}$

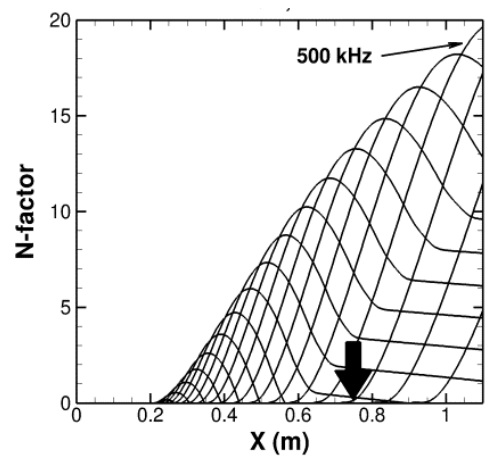

(d) $t=21 \mathrm{~s}$

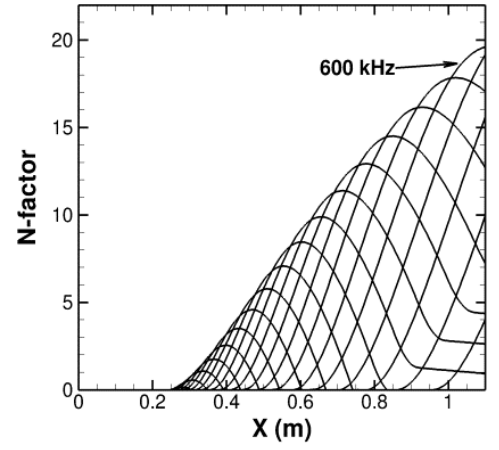

(b) $t=19 \mathrm{~s}$

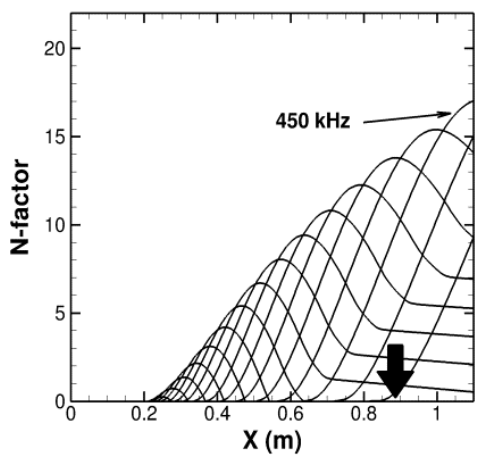

(e) $t=22 \mathrm{~s}$

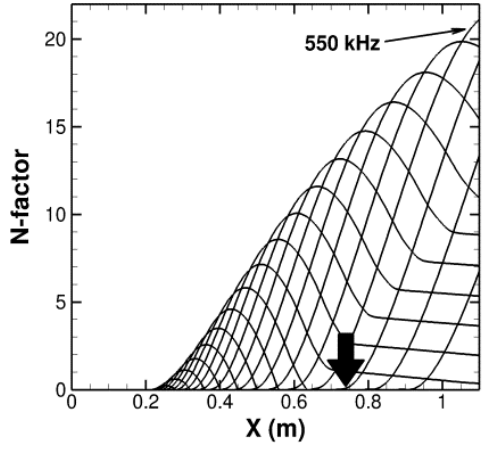

(c) $t=20 \mathrm{~s}$

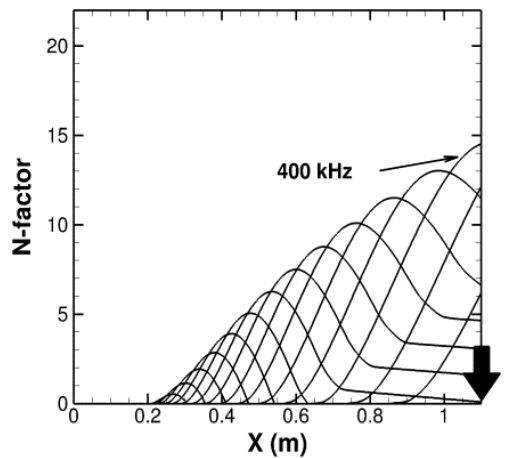

(f) $t=23 \mathrm{~s}$

Figure 4. $\mathrm{N}$-factor evolution for second mode disturbances of various frequencies from $t=18$ to 23 seconds. The disturbance frequency decreases by $25 \mathrm{kHz}$ across each adjacent pair of $\mathrm{N}$-factor curves. The black arrows indicate transition locations.

\section{HIFiRE-1 Transition Analysis during Re-entry Phase}

As described in Refs. 3 and 4, the angle of attack during the re-entry phase of HIFiRE-1 flight departed substantially from the design value of zero degrees. The instrumentation pattern for the HIFiRE- 1 model was designed to provide detailed 2D maps of surface temperature and/or heat flux over the majority of the cone surface. However, as a byproduct of the spinning of the (axisymmetric) cone model during flight, information regarding the crossflow transition behavior could be obtained from high-frequency pressure and heat transfer transducers as they transited the transition front. The analysis of transition based on the data from thermocouples and heat transfer gauges was complicated by the large temporal variations in the angle of attack as well as the drift in thermocouples prior to transition onset during the reentry phase ${ }^{3}$. However, valuable information was gained from high-frequency pressure measurements, especially those obtained via the pressure transducer PHBW1 at $X=0.85 \mathrm{~m}$. The findings from Refs. 3 and 4 were used to select specific flow conditions for computational analysis as listed in Table I.

As described in Ref. 5, at higher amplitude, quasi-periodic surface pressure fluctuations were first discernable within the time history of the PHBW1 signal at approximately 481.11 seconds. The frequency of these disturbances (or, effectively, an azimuthal wavenumber based on the cone roll rate) could be visually estimated using the signal window between 481.25 seconds and 481.26 seconds. Accordingly, the trajectory point corresponding to $t=481.3$ seconds was selected to ensure that the computed basic flow would support a measurably strong stationary crossflow instability, with due allowance for the effects of the significant uncertainty in the angle of attack $\alpha$. The data analysis also 
indicated the first turbulent signal along the windside ray at $t=484.25$ seconds. The last identifiable quasi-periodic fluctuations prior to the breakdown to turbulence were observed within the crossflow region at approximately $t=484.58$ seconds. At $t=485$ seconds (condition R3 from Table I), the flow at $X=0.85 \mathrm{~m}$ was believed to be turbulent at all azimuthal locations. The flow conditions corresponding to R4 were derived from an earlier version of the flight trajectory and the angle of attack was based on a smoothing of the raw angle of attack estimates based on surface pressure measurements. The differences between cases R3 and R4 illustrate the increased uncertainty in flow conditions during the re-entry phase. The intermediate trajectory point R 2 at $t=483.7$ seconds was selected for computational analysis for two specific reasons: (i) a Reynolds number value that is approximately halfway between the two extrema corresponding to flow conditions R1 and R3, and (ii) its close proximity to the trajectory point where the periodic pressure fluctuations (normalized by the local pressure) are at a maximum.

Table I. Freestream conditions at selected times during descent phase.

\begin{tabular}{|l|l|l|l|l|l|l|l|}
\hline $\begin{array}{l}\text { Flow } \\
\text { Condition }\end{array}$ & $\begin{array}{l}\text { Time } \\
(\mathrm{s})\end{array}$ & $\begin{array}{l}\alpha \\
(\mathrm{deg})\end{array}$ & $\begin{array}{l}\mathrm{P}_{\infty} \\
(\mathrm{Pascal})\end{array}$ & $\begin{array}{l}\mathrm{T}_{\infty} \\
(\mathrm{K})\end{array}$ & $\mathrm{M}_{\infty}$ & $\begin{array}{l}R e \\
\left(10^{6} / \mathrm{m}\right)\end{array}$ & $\begin{array}{l}\text { Altitude } \\
(\mathrm{km})\end{array}$ \\
\hline $\mathrm{R} 1$ & 481.3 & 13.60 & 1126.975 & 230.508 & 6.931 & 2.40 & 30.611 \\
\hline $\mathrm{R} 2$ & 483.7 & 9.60 & 2278.999 & 223.175 & 6.974 & 5.10 & 25.864 \\
\hline $\mathrm{R} 3$ & 485.0 & 7.50 & 3317.379 & 216.258 & 7.030 & 7.80 & 23.426 \\
\hline R4* & 485.0 & 6.14 & 3491.140 & 217.886 & 7.196 & 8.32 & 23.461 \\
\hline * : Earlier version of flight trajectory, angle of attack based on smoothing of raw data \\
\hline
\end{tabular}

The flow configuration R4 also resembles the ground test configuration for quiet tunnel experiments at Purdue University in terms of the cone half-angle and the angle of attack ${ }^{11}$, which supports second mode instabilities along the windward and leeward lines and strong crossflow instabilities in between. While the model surface temperatures during the ground experiment are moderately lower than those in the HIFiRE-1 flight experiment, the freestream static temperature is considerably lower than that in flight. Because of the considerably higher value of the ratio of model surface temperature to adiabatic surface temperature in the ground experiment, that configuration also supports modest amplification of first mode waves $^{12}$, which is not anticipated in the flight case.

This paper outlines the computational analysis pertaining to the trajectory point $\mathrm{R} 1$ at $t=481.3$ seconds, which highlights the effects of a moderately high angle of attack (the ratio of angle of attack to cone halfangle is 1.94) on the basic state as well as the flow instability characteristics. A brief set of results pertaining to stability predictions for the flow condition $\mathrm{R} 4$ are also included. The analysis of crossflow instabilities at $t=481.3$ seconds and $t=485$ seconds should also provide an opportunity for a preliminary comparison between the linear stability based transition correlation for the HIFiRE-1 model with the earlier findings for the Pegasus flight experiment ${ }^{13}$. More comprehensive analysis of the transition behavior during the reentry phase including the flow conditions R2 and R3 is deferred to a future paper. 


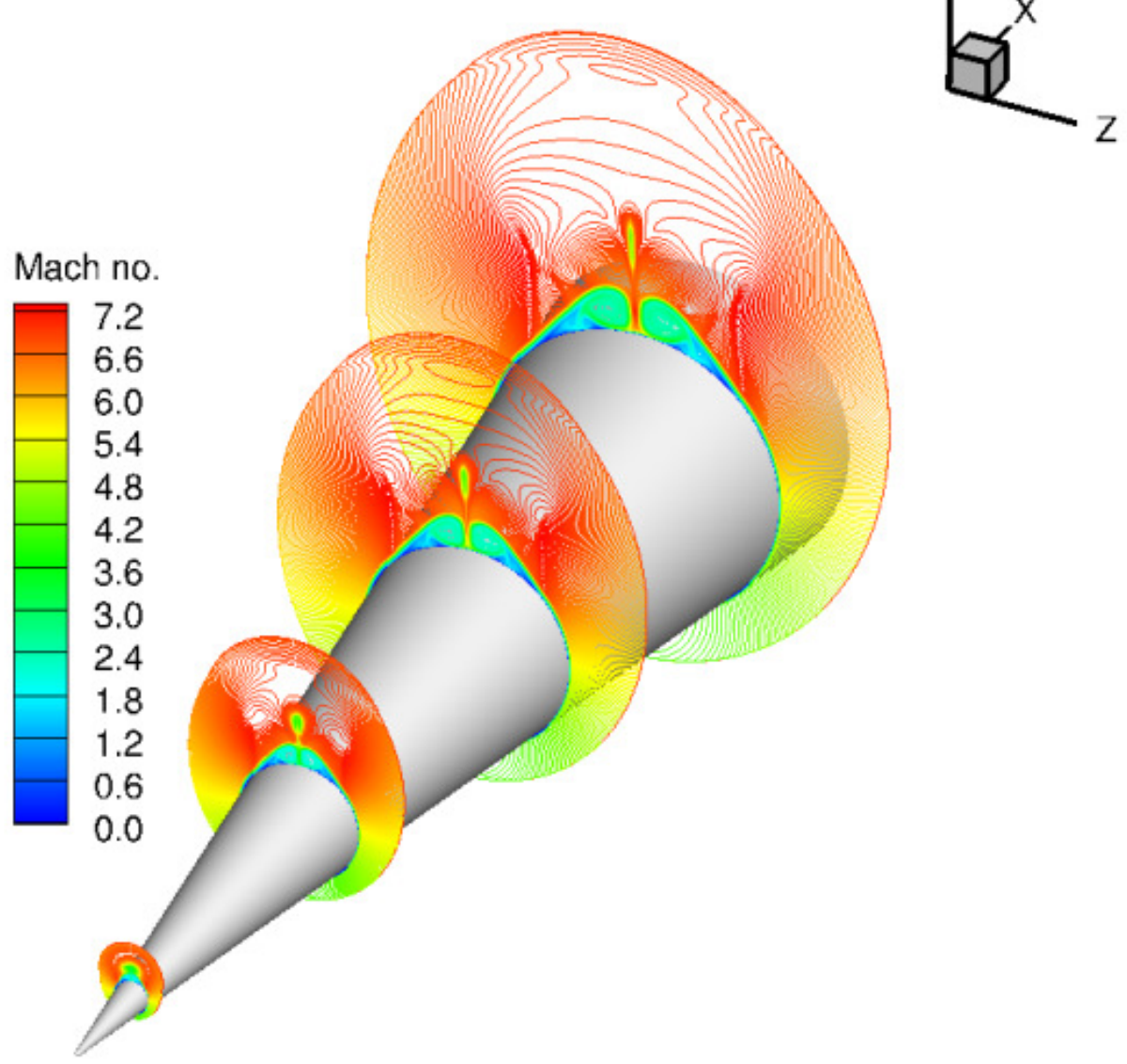

Figure 5. Mach number contours at selected axial locations for case R1

Because of drift issues with a number of surface thermocouples during the exo-atmospheric segment of the trajectory, the surface temperature distributions used for the mean flow computation pertaining to the reentry cases were based entirely upon the thermal analysis using the TOPAZ code. It was assumed that no unsteady vortex shedding occurs at the flow conditions of interest and, therefore, that the (undisturbed) laminar basic state is purely stationary and, furthermore, symmetric with respect to both windward and leeward planes of symmetry.

Figure 5 presents an overview of the basic state at $t=481.3$ seconds in the form of Mach number contours at selected axial locations. Unlike the cone in the flight experiment, the computational cone does not spin. Given the slow spinning rate of $4 \mathrm{~Hz}$ or so in the flight experiment, exclusion of the spinning effect is an acceptable simplification and avoids a doubling of the required computational resources. The flow contours at the downstream three stations are qualitatively similar. They are suggestive of a separation of the secondary flow near $\theta=180 \pm 41$ degrees, where $\theta$ is the cone azimuthal angle measured from the windward meridian, followed by a roll up between the separation location and the leeward plane of symmetry. The presence of crossflow separation over cones at relatively large angles of attack is wellknown in the literature and has been confirmed in both experiments ${ }^{14}$ and computations based on a parabolic approximation to the Navier-Stokes equations ${ }^{15}$. The roll-up of the secondary flow appears to cause the near pinching of an inverted tear drop shaped structure centered on the leeward meridian. This structure consists of relatively slow moving fluid that continues to lift away from the surface at increasing $X$ locations, possibly as a result of self-induced velocity by the counter-rotating pair of crossflow- 
separation vortices. Analogous features have been observed in steady state computations of laminar flow behind an isolated roughness element ${ }^{16}$. The above-mentioned structure is nearly circular at the farthest upstream location, but becomes increasingly oblong over the length of the cone.

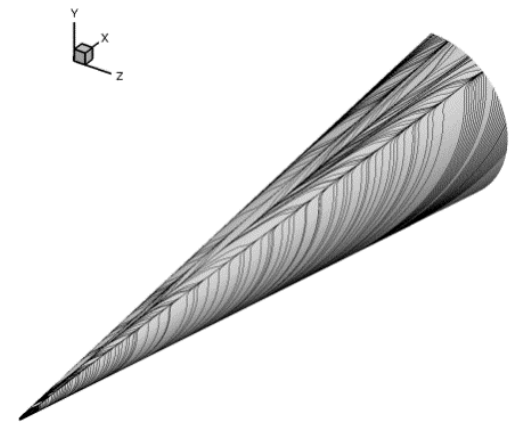

(a) Overall

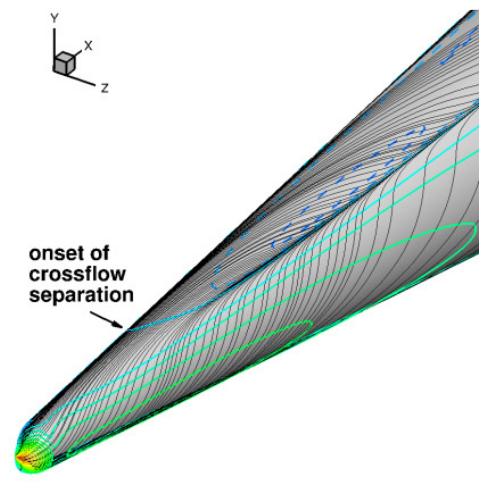

(b) Close-up view near nose

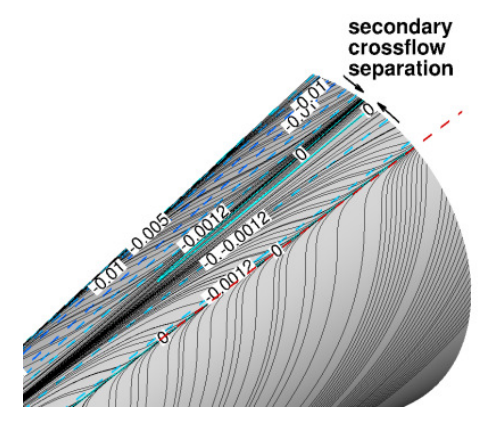

(c) Close-up view near end of cone

Figure 6. Limiting surface streamlines for case $\mathrm{R} 1(t=481.3$ seconds). Top boundary of each image corresponds to leeward line.

The presence of crossflow separation is confirmed by the limiting surface streamlines as shown in Fig. 6(a). These streamlines also confirm the nearly conical behavior of the separated crossflow, except in the vicinity of the nose where the onset of crossflow separation occurs (Fig. 6(b)). A close up view of the surface streamlines near the end of the cone (Fig. 6(c)) provides a hint of a more complex structure underneath the large scale roll-up, perhaps in the form of secondary vortices. The circumferential variations in both the azimuthal shear stress and the total shear stress at selected axial stations are shown in Figs. 7(a) and 7(b), respectively. In addition to confirming the conical behavior of the crossflow separation characteristics, Fig. 7(a) also confirms the existence of an inner region of secondary separation with positive values of azimuthal shear stress.

Despite the finite nose radius (with a nose radius based Reynolds number of approximately 6,000), a nonuniform surface temperature distribution, and the presence of crossflow separation, the axial velocity profiles within the boundary layer along the windward plane of symmetry are nearly self-similar as seen from Figs. 8(a)-(b). Velocity profiles along the leeward line do not exhibit self-similarity and the heat flux and wall shear distributions do not follow an $X^{-1 / 2}$ decay along the symmetry line. Circumferential variation of streamwise, crossflow and wall-normal velocity profiles at $X=0.85 \mathrm{~m}$ is shown in Figs. 9(a) through 9(c), respectively. As may be expected, Fig. 9(b) indicates a reversal in the crossflow direction beyond the onset of crossflow separation. The roll-up of the separated secondary flow is correspondingly manifested in a change in the sign of the wall-normal velocity component (Fig. 9(c)).

Given the relatively strong spanwise variations in the vicinity of the leeward meridian, a conventional stability analysis of the velocity profiles along the leeward plane is not meaningful. However, it is appropriate to perform such stability analysis for the boundary layer flow along the windward plane of symmetry. $\mathrm{N}$-factor curves for axisymmetric second mode disturbances at various frequencies are shown in Fig. 10 for cases R1 and R2. The peak $\mathrm{N}$-factor values for case $\mathrm{R} 1$ at $X=0.85 \mathrm{~m}$ (the location of the PHBW1 transducer) and over the entire length of the cone are approximately 4.8 and 6.4, respectively (Fig. 10 a). Based on the correlations derived from the ascent phase analysis, transition onset along the windward plane is not expected at $t=481.3$ seconds. On the other hand, for case R 2 at $t=483.7$ seconds, the boundary layer flow within the windward plane is highly unstable to second mode disturbances and the $\mathrm{N}$-factor values at $X=0.85 \mathrm{~m}$ and $X=1.05 \mathrm{~m}$ (near the end of the cone) reach 14 and 17 , respectively 
(Fig. $10 \mathrm{~b}$ ). This finding is consistent with the flight data analysis ${ }^{5}$, which had indicated the onset of transition at $X=0.85 \mathrm{~m}$ along the windward line to be at $t \approx 483.8$ seconds.

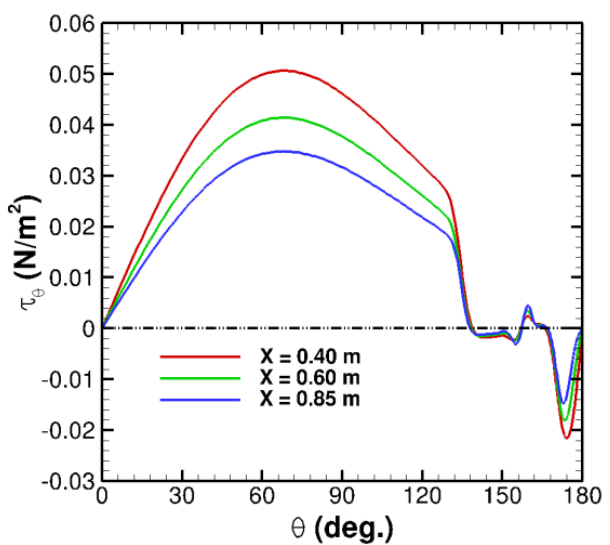

(a) Azimuthal shear stress

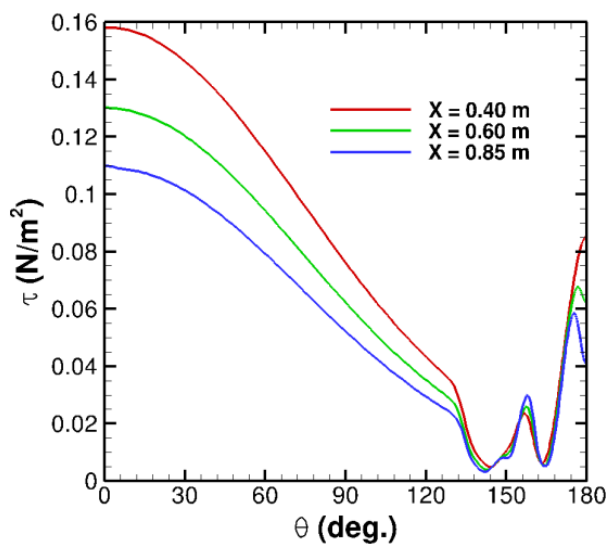

(b) Total shear stress

Figure 7. Circumferential variation in surface shear stress at selected axial stations.

The analysis of crossflow instabilities away from the symmetry planes is considered next. The time history of pressure disturbances measured by the high-frequency transducer PHBW1 is suggestive of the presence of stationary crossflow instability modes with surface pressure disturbance levels of approximately 3.5 percent of the free-stream static pressure ${ }^{4}$. A classical stability analysis was performed for stationary disturbances of fixed azimuthal wavenumber ranging from 20 to 200 . The spatial amplification rate is integrated along various trajectories that are aligned with the streamlines outside the boundary layer. Fig. 11 (a) illustrates the comparison between the inviscid streamline pattern and the limiting surface streamlines presented earlier in Fig. 6. Contours of the peak N-factor value (i.e., maximized over all azimuthal wavenumbers) as a function of location over the cone surface are plotted in Fig. 11(b). It is seen that, for $X=0.85 \mathrm{~m}$, the highest $\mathrm{N}$-factor values are observed within a narrow band of azimuthal locations near $\theta=102$ degrees relative to the windward meridian. The associated $\mathrm{N}$-factor value is between 9 and 10. For the Pegasus flight experiment, the onset of crossflow transition was correlated with stationary $\mathrm{N}$-factors (computed using group velocity trajectories) between the range of 7 and $12.4^{13}$. However, the comparable $\mathrm{N}$-factor value in the present case is not necessarily inconsistent with the inferred absence of transition in the experiment. That is because boundary layer transition due to stationary crossflow modes is highly sensitive to the external disturbance environment, particularly the surface finish characteristics. Crossflow amplification factors exceeding $\mathrm{N}=12$ have been noted in subsonic flight experiments with smooth surfaces ${ }^{17}$. Under certain ambient conditions, the traveling crossflow instability may also play a significant role in transition, which is currently being investigated as part of ongoing work. Finally, the substantial uncertainty in descent angle of attack would also have an effect on the $\mathrm{N}$-factors and an attempt is being made to reduce that uncertainty as well. 


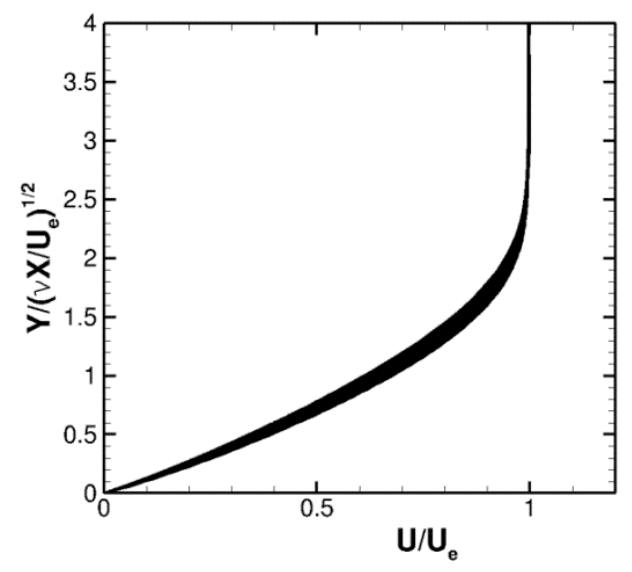

(a) $0.4 \mathrm{~m}<X<0.6 \mathrm{~m}$

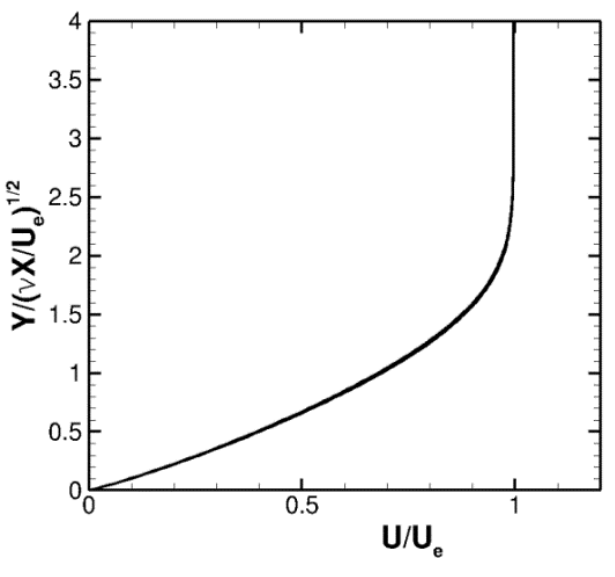

(b) $X>0.6 \mathrm{~m}$

Figure 8. Axial velocity profiles at various stations along windward meridian.

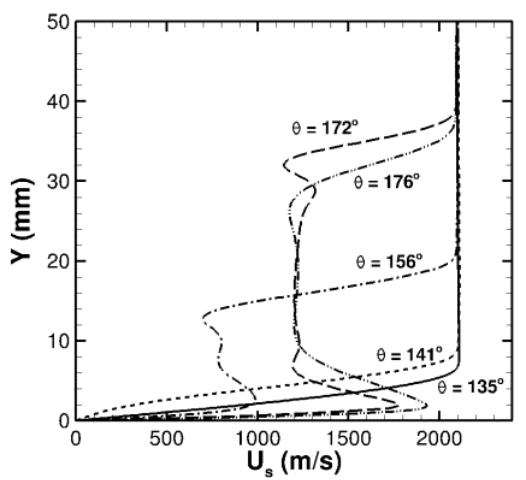

(a) Streamwise velocity

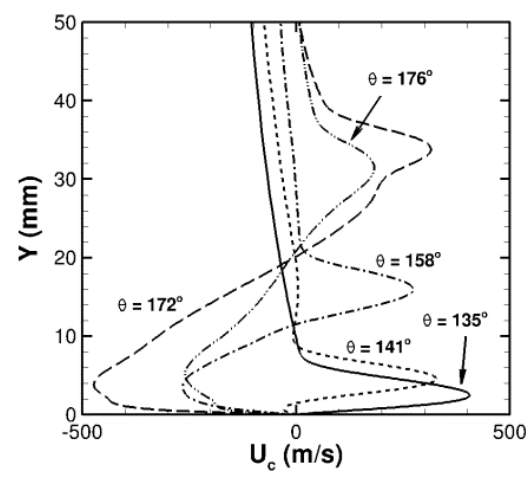

(b) Crossflow velocity

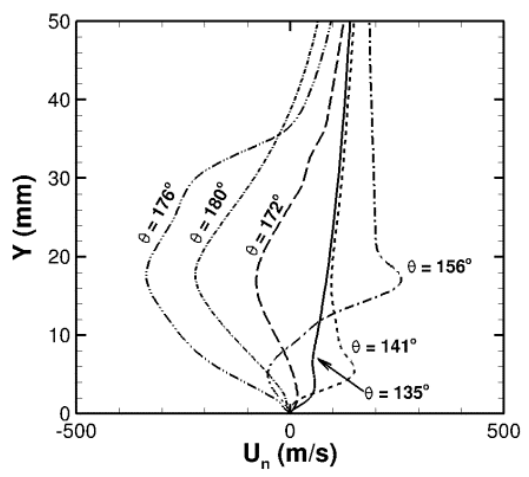

(c) Wall-normal velocity

Figure 9. Velocity profiles at $X=0.85 \mathrm{~m}$.

The range of azimuthal wavenumbers that are most amplified at $X=0.85 \mathrm{~m}$ corresponds to $n \approx 60-80$. Based on Fig. 12 from Ref. 4, one estimates that the quasi-periodic pressure disturbances recorded by the PHBW1 transducer corresponds to an azimuthal wavenumber of $n \approx 80$ for a cone spinning rate of 4 cycles per second and a wavenumber of approximately $n \approx 90$ for a spinning rate of 3.5 cycles per second. The unsteady pressure data from the PHBW1 transducer at $X=0.85 \mathrm{~m}$ revealed a transition front centered within the region of strong crossflow instability from Fig. 11(b). However, the inferred location of forward-most transition was found to be at an azimuthal location of $\theta \approx 75$ degrees, which is noticeably different from the center of peak $\mathrm{N}$-factor values at $X=0.85 \mathrm{~m}(\theta \approx 102$ degrees $)$. This discrepancy could, in part, be caused by the ad hoc approximations required to compute crossflow $\mathrm{N}$-factors in an azimuthally varying boundary layer flow, as well as uncertainties in angle of attack. In particular, we note that the propagation of crossflow disturbances in azimuthally inhomogeneous boundary layers is strongly dependent on a sufficient knowledge of the source of the instability field. Yet, the ad hoc analysis has provided useful information regarding the strength of instability amplification and the frequency-wavelength characteristics of the dominant instability modes. 


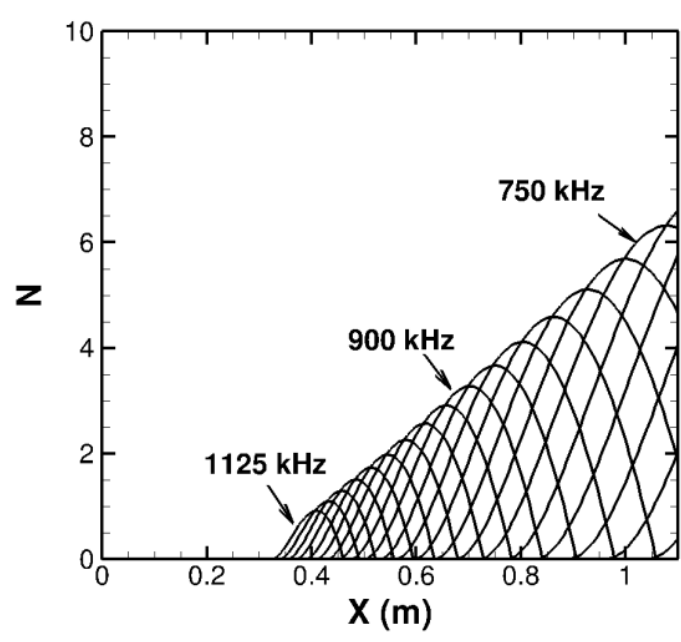

(a) Case R1.

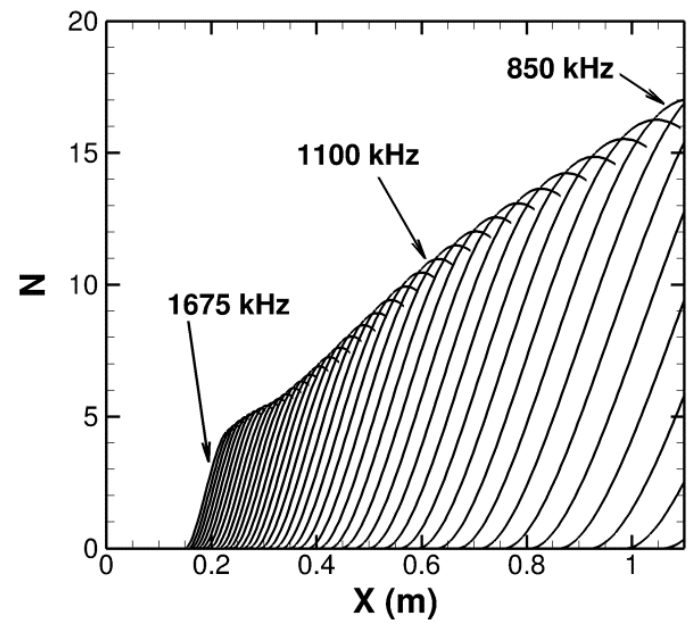

(b) Case R2

Figure 10. N-factor curves for axisymmetric second mode disturbances at various frequencies along major axis symmetry line.

For the preliminary stability analysis presented herein, the streamlines in the inviscid region are chosen as the integration trajectories to determine the amplification ratio of relevant instability modes.

Instability amplification characteristics for the case R4 are examined next. Due to the lower angle of attack, the secondary flow in this case is significantly different from that in case R1 as shown by the Mach number contours in Fig. 12(a). The second mode $\mathrm{N}$-factors along the windward line reach 14 just past the midway point of the cone and approach 23 near the end of the cone. The $\mathrm{N}$-factors along the leeward line are considerably smaller, only about 9.5 . This weaker second mode instability along the leeward plane is attributed to the substantially modified local mean flow as a result of the convergence of secondary flow from either side of this plane ${ }^{7}$. Because of the finite angle of attack, crossflow instability is, again, important away from the windward and leeward planes. Figure 12(b) shows the N-factor contours for stationary crossflow instability, obtained as an envelope of the amplification curves for stationary modes of fixed azimuthal wavenumber. The $\mathrm{N}$-factor reaches a value of 14 just before $X=0.5$ $\mathrm{m}$. The N-factor values shown in Fig. 12 are capped off at 14 to emphasize that crossflow transition is likely to occur over the middle portion of the cone, but the actual $\mathrm{N}$-factors reach a maximum of approximately 26 at the end of the cone. The large $\mathrm{N}$-factors for the R4 case are consistent with the finding from the flight measurements that the flow over the aft portion of the cone is turbulent by $t=485$ seconds. 


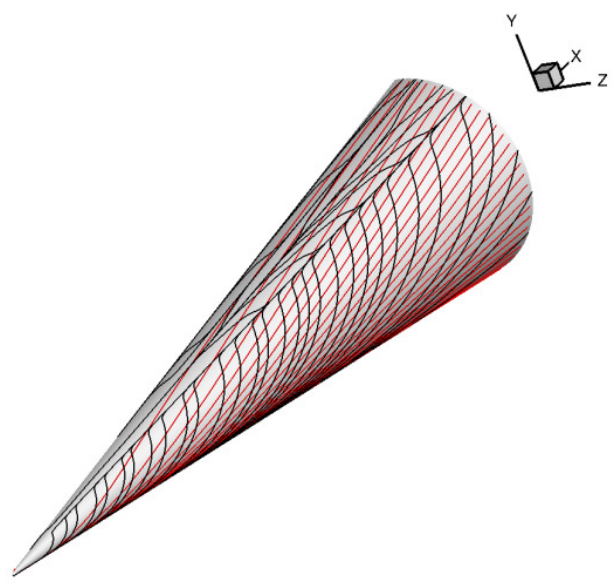

Fig. 11(a). Inviscid streamline pattern (red curves) used for $\mathbf{N}$-factor calculation along with limiting surface streamlines (black curves).

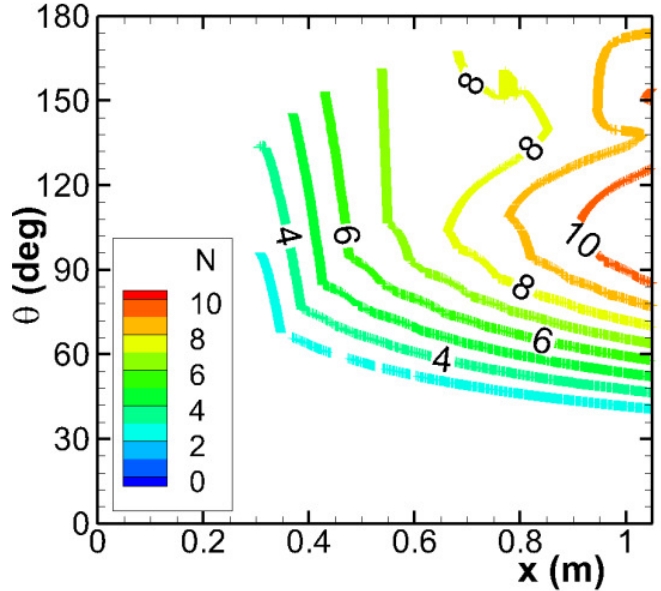

Fig. 11(b). N-factor contours for stationary crossflow mode disturbances. Bottom of the figure corresponds to windward side and the top boundary is leeward side.

Figure 11. Analysis of stationary crossflow instability for trajectory point $R 1$ at $t=481.3$ seconds

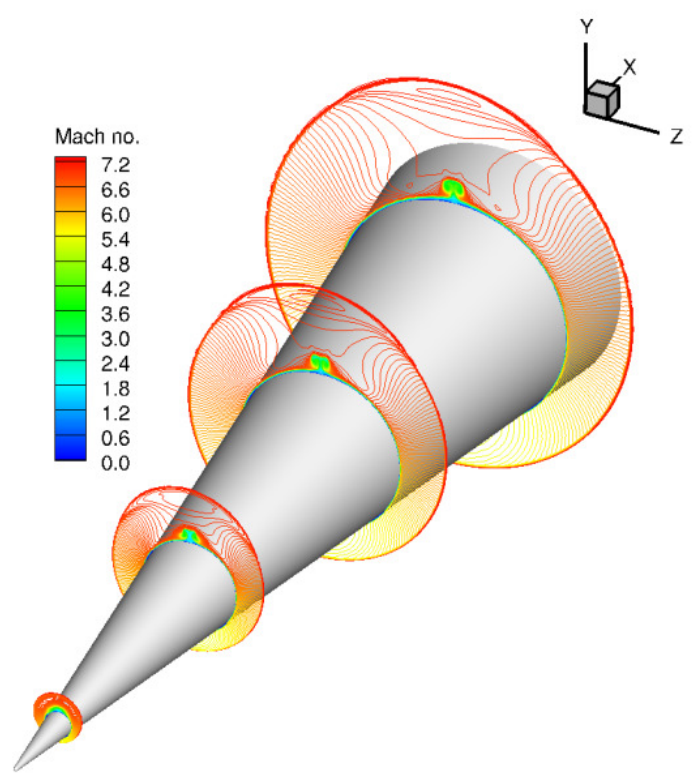

Fig. 12(a). Mach number contours at selected axial locations.

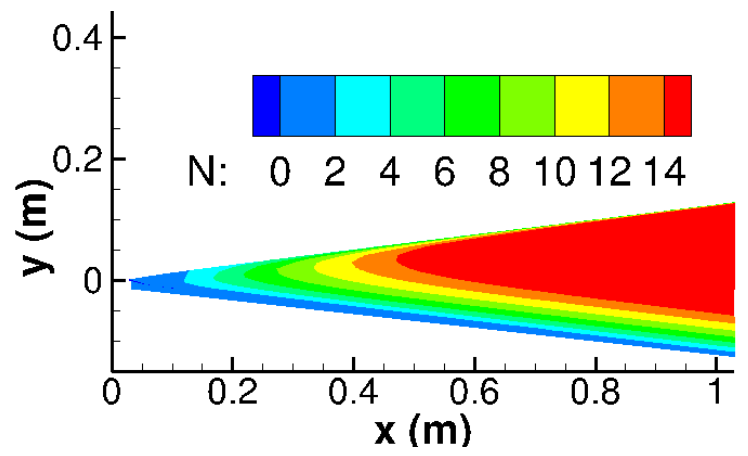

Fig. 12(b). $\mathrm{N}$-factor contours for stationary crossflow mode disturbances. Bottom of the cone is windward side and top is leeward side.

Figure 12. Flow field and stability analysis for trajectory point R4. 


\section{Stability Characteristics of Scaled HIFiRE-5 Configuration and Comparison with Measurements}

Results for the HIFiRE-5 configuration (a blunt nosed elliptic cone with 2:1 ellipticity) are presented in this section. The computations are based on a wind tunnel model of $38.1 \%$ the size of the full scale configuration designed for the flight test. Details of the model in relation to the full scale cone can be found in references 8 and 19. The $38.1 \%$ scale wind tunnel model is 328 millimeters in length. The major and minor axes of the elliptical cross-section at the cone base are, respectively, 164 and 82 millimeters. The cone half angles are 13.797 and 7 degrees along the major and minor axes, respectively. The computations presented in this paper are based on the same configuration, only that the length of the cone is extended to 381 millimeters. Given the high Mach number, the upstream influence of perturbations is negligible. Hence, the flow over first 328 millimeters of the computational cone should correspond exactly to that over the experimental model.

The mean boundary layer flow over the cone surface was computed using the VULCAN code, similar to the HIFiRE-1 case discussed in the previous sections. Two separate Reynolds number cases were considered and the flow conditions for these two cases are listed in Table II. Condition 1 is identical to that considered by Choudhari et al. ${ }^{16}$, whereas condition 2 corresponds to one of the cases for which unsteady pressure measurements were recently performed by Borg et al. ${ }^{18}$

Table II. Flow conditions for HIFiRE-5 elliptic cone

\begin{tabular}{|l|l|l|l|l|l|l|l|}
\hline Case & $\mathrm{M} \infty$ & $\operatorname{Re}(1 / \mathrm{m})$ & $\rho_{\infty}\left(\mathrm{kg} / \mathrm{m}^{3}\right)$ & $\mathrm{T} \infty(\mathrm{K})$ & $\mathrm{P} \infty(\mathrm{kPa})$ & $\mathrm{U} \infty(\mathrm{m} / \mathrm{s})$ & $\mathrm{T}_{\mathrm{w}}(\mathrm{K})$ \\
\hline 1 & 6 & $9.843 \times 10^{6}$ & 0.04034 & 52.8 & 0.611 & 873.92 & 300 \\
\hline 2 & 6 & $8.275 \times 10^{6}$ & 0.03121 & 49.9 & 0.447 & 849.84 & 300 \\
\hline
\end{tabular}

The mean flow solutions used in Ref. 8 had evidence of streamwise elongated streaks in both surface flow visualizations based on wall shear and heat flux distributions, as well as in the streamwise velocity distribution at a fixed axial station. The number and intensity of the streaks increased with flow Reynolds number. However, given the focus of that study on providing pre-flight guidance related to transition behavior on the HIFiRE-5 configuration, a definitive study to establish both the existence and the character of the secondary vortices beyond numerical uncertainty could not be performed at that time. Such computations were performed soon after the completion of the study in Ref. 18 and the results of those are included herein as a prelude to the study of Case 2 from Table II and the associated comparison with the measurements by Borg et al. ${ }^{18}$.

The grid convergence analysis for Case 1 used five grids with successively increasing resolutions. The detailed grid resolutions are listed in Table III. The grid was refined uniformly in both surface directions, so that the medium and coarse grids could be obtained from the fine grid by using every $2^{\text {nd }}$ and $4^{\text {th }}$ point, respectively, in both directions tangential to the surface. The finer grid has the same resolution along the surface as the fine grid, but double the resolution in the wall-normal direction, whereas the finest grid corresponds to a two-fold refinement of the fine grid all three directions. Thus, the surface grid refinement from coarse to medium to fine to the finest grid is fully characterized by a single scalar parameter in the form of the ratio of the total number of cell faces relative to some fixed reference or, equivalently, by the increase in spatial resolution along either of the two surface directions. Based on extensive experience with applying the VULCAN flow solver to slender body configurations at modest angles of attack, the wall-normal resolution corresponding to all grids from Table III is deemed to be satisfactory. To confirm the adequacy of the wall normal resolution, the finer and finest grids also include twice the number of grid cells along the wall normal direction. Each of the solutions from the grid resolution study was converged to machine zero.

14

American Institute of Aeronautics and Astronautics 
Figures 13(a) and 13(b) compare the computed velocity profiles at selected streamwise stations across the length of the elliptic cone along the major and minor axis meridians, respectively. Except for the profiles based on the two coarsest grids, which show obvious yet modest differences with the others (especially at the farther downstream stations), the results based on the three grids with higher resolutions cannot be visually distinguished from each other. Figures 13(c) and 13(d) compare the profiles of the product of flow density and the normal-direction derivative of streamwise velocity, which we term the specific angular momentum. The results are similar to those for the streamwise velocity profiles in Figs. 13(a) and 13(b). Thus, grid convergence is established in these metrics along the two boundaries of the computational domain. Extensive prior experience with this and other related configurations with varying degrees of nose bluntness has shown that, at least for moderate angles of attack (i.e., prior to the onset of crossflow separation), the solution in the vicinity of the minor axis plane of symmetry leeward plane (for elliptic cone) and near the leeward plane of symmetry (for axisymmetric configurations at moderate angle of attack ${ }^{20,21}$ ) tends to be the most sensitive to grid resolution.

Table III. Grid resolutions for convergence test

\begin{tabular}{|c|c|c|c|c|}
\hline $\begin{array}{c}\text { Grid } \\
\text { designation }\end{array}$ & $\begin{array}{c}\text { Total cells in } \\
\text { axial direction } \\
\text { downstream of } \\
\text { the nose region }\end{array}$ & $\begin{array}{c}\text { Total azimuthal } \\
\text { cells at a fixed } \\
\text { axial station }\end{array}$ & $\begin{array}{c}\text { Total wall- } \\
\text { normal cells }\end{array}$ & $\begin{array}{c}\text { Total number of } \\
\text { cells (Million) }\end{array}$ \\
\hline Coarse & 150 & 90 & 352 & 5.456 \\
\hline Medium & 300 & 180 & 352 & 21.824 \\
\hline Fine & 600 & 360 & 352 & 87.296 \\
\hline Finer & 600 & 360 & 704 & 174.592 \\
\hline Finest & 1200 & 720 & 704 & 698.368 \\
\hline
\end{tabular}

Figure 14 presents the surface streamlines (friction lines) as well as Mach contours on the outflow and selected axial planes cut though the flow solution. The surface streamlines and axial planes on the initial $20 \%$ of the body show low momentum boundary layer fluid being pushed toward the vertical plane of symmetry, resulting in a thickening of the boundary layer due to the convergence of flow from both sides of the plane of symmetry. The next-to-last plane shows that this bulge eventually rolls up into classical vortical structure with a cross flow separation line being evident in the surface streamlines. However the azimuthal pressure gradient continues to push boundary layer fluid azimuthally towards the centerline as the flow moves axially until that flow encounters the blockage created by the centerline vortex. Between the last two planes, a second bulge immediately outboard of the centerline vortex are is seen to be developing. As in the case of the centerline vortex this bulge eventually rolls up into a second vortex and so on. Thus, at a fixed Reynolds number, the strength as well as the number of vorticity structures increases along the downstream direction as mentioned at the beginning of this section. Equivalently, on expects a similar trend at a fixed axial station when the unit Reynolds number is increased. Although not shown here, the images analogous to Fig. 14 but based on the fine and finest grids are visually identical to Fig. 14. Even for the coarse and medium grids, the locations and the extent of roll-up associated with the vortex structures are the same, except for the contours at the downstream locations being slightly diffused in comparison to those in Fig. 14. 


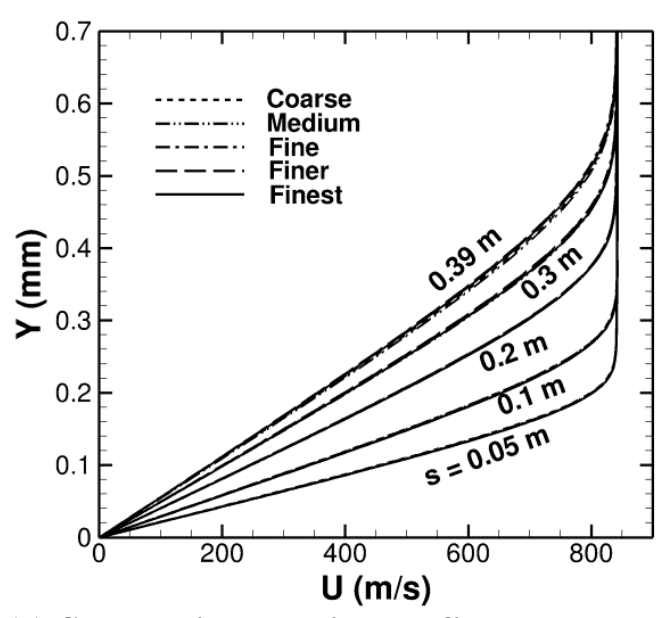

(a) Streanwise velocity profiles along major axis meridian (attachment line).

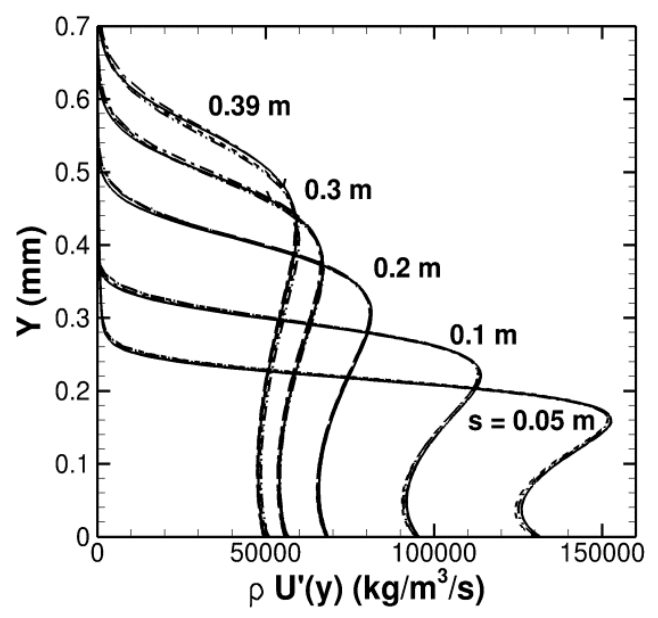

(c) Specific angular momentum profiles along major axis meridian. Line legends same as in (a) above.

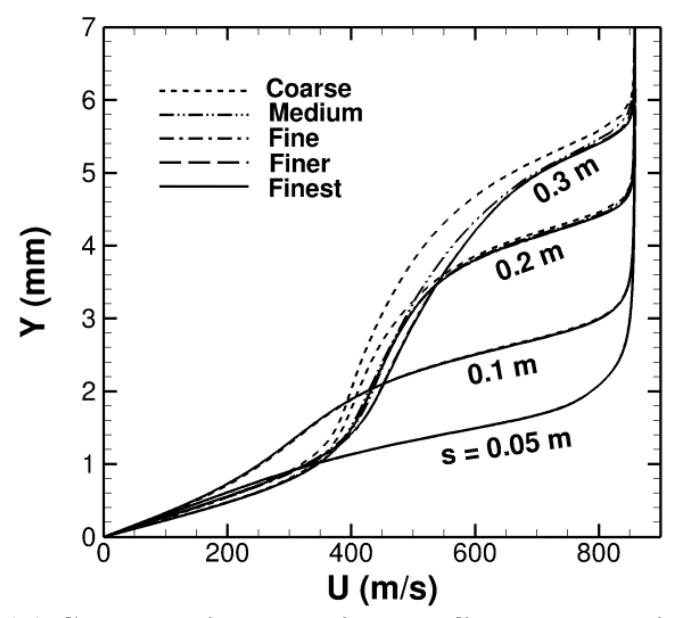

(b) Streamwise velocity profiles along minor axis meridian.

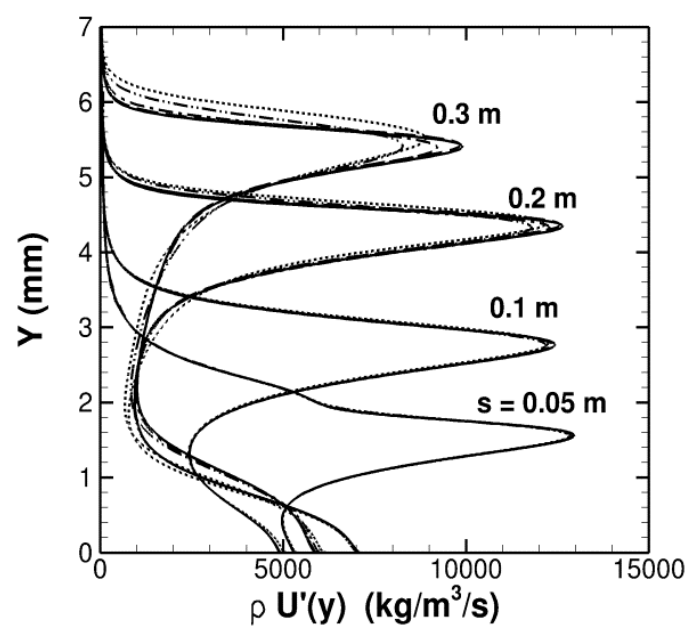

(d) Specific angular momentum profiles along minor axis merdian. Line legends same as in (b) above.

Figure 13. Streamwise velocity and specific angular momtum profiles for various grid resolutions for HIFiRE-5 elliptic cone at selected surface distance, $s$, along respective meridians.

Fig. 14 also shows that the nature of vortex roll-up, especially for the secondary vortex structures that reside in the region of nonzero crossflow, is qualitatively similar to the crossplane contours of finite amplitude stationary crossflow instabilities. In a realistic flow environment, such stationary crossflow instabilities would be excited, for instance, by surface geometry variations that overlap, in wave number spectrum, with the stationary crossflow modes. We believe that the main differences between that case and the idealized problem focused exclusively on the base flow computation as described in this section are as follows: The stationary vortex structures in Fig. 14 represent the intrinsic (and probably generic for the class of flows mentioned herein) dynamics of the 3D boundary layer and, therefore, do not require any external excitation. Thus, the locations of these vortices (i.e., the vortex spacing) as well as their amplitude evolution are determined entirely by the underlying body shape and the flow conditions and not 
by the surface roughness characteristics or by the numerical noise. As such, the basic state involves short scale azimuthal variations that must be considered in predicting the evolution of small amplitude disturbances excited via external means. Furthermore, the streaky structures in surface flow visualizations based on heat transfer distribution (measured via temperature sensitive paint or infra-red or phosphor thermography, for instance) would include the contributions from both intrinsic and externally excited streaks.

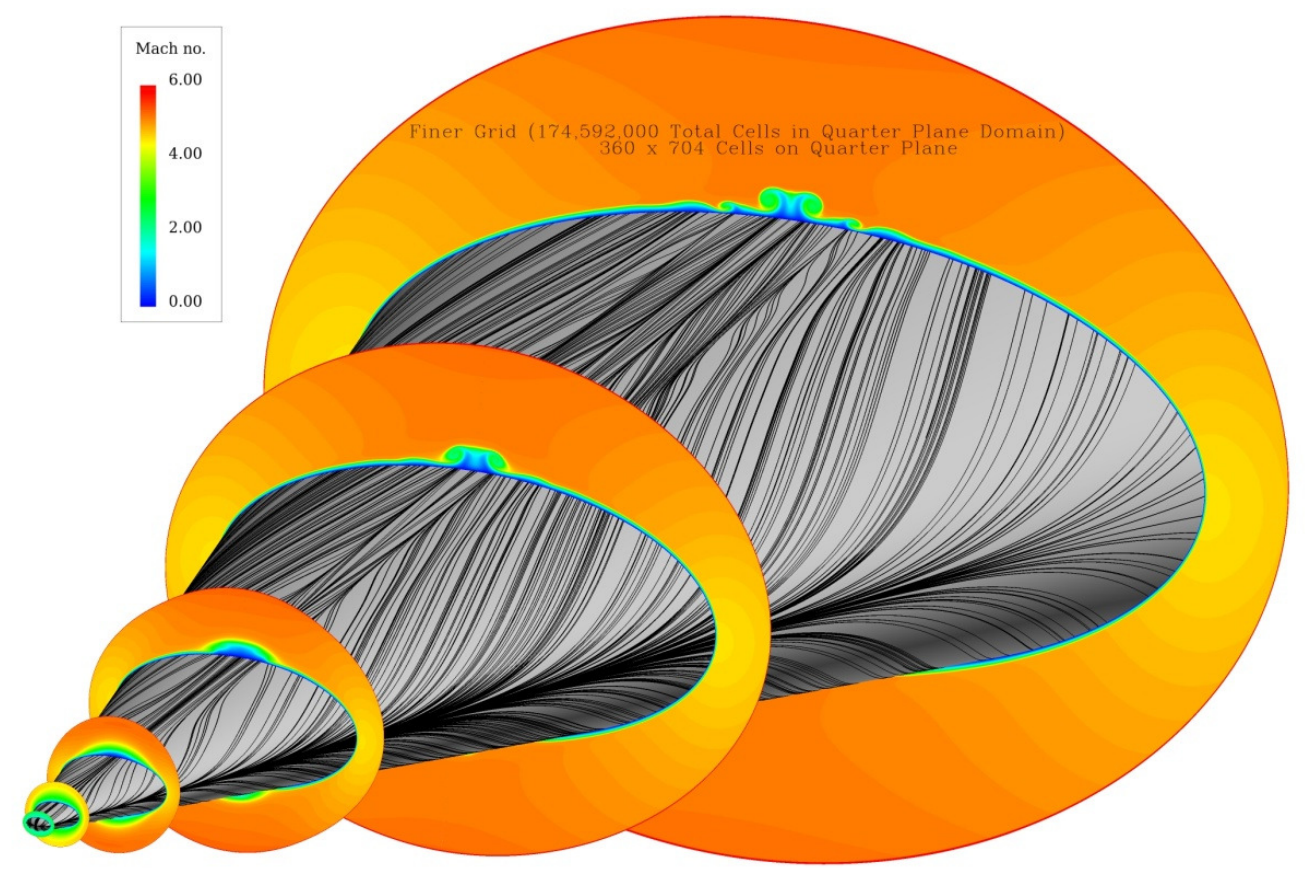

Figure 14. Mach number contours at different cross-sections for HIFiRE-5 elliptic cone (Case 1 from Table II).

Specifically targeted experimental measurements combining a highly polished model surface (to minimize extraneous, roughness induced vortex structures) and accurate, well-resolved off-body flowfield measurements analogous to performed by Kegerise et al. ${ }^{22}$ behind a discrete roughness element in a lower Mach number flow would provide a definitive confirmation of the computationally observed streaks associated with an undisturbed laminar basic state.

Classical stability analysis is used to examine the growth of second mode and crossflow instabilities for condition 2 from Table II using the mean flow solution obtained with the fine grid. Second mode $\mathrm{N}$-factor curves are show in Figs. 15(a) and 15 (b) and along the major axis meridian (attachment line) and the minor axis meridian, respectively. The attachment line is very stable with a maximum second mode $\mathrm{N}$ factor of less than 1 (Fig. 15(a)), indicating a laminar attachment line. Indeed, the measurements of Borg et al. ${ }^{18,19}$ show that the attachment line is laminar even under noisy conditions unless it is tripped. On the other hand the flow along the minor axis meridian is highly unstable, despite the decrease in unit Reynolds number relative to the PUa0b0R3 case from ${ }^{8}$, and the maximum value of second mode Nfactor is approximately 10 (Fig. 15(b)). Of course, because of the rapid azimuthal variations associated with the primary vortex roll-up near the leeward line, accurate predictions of instability evolution along this plane may well require an advanced stability analysis based on partial differential equations ${ }^{23,24}$. 


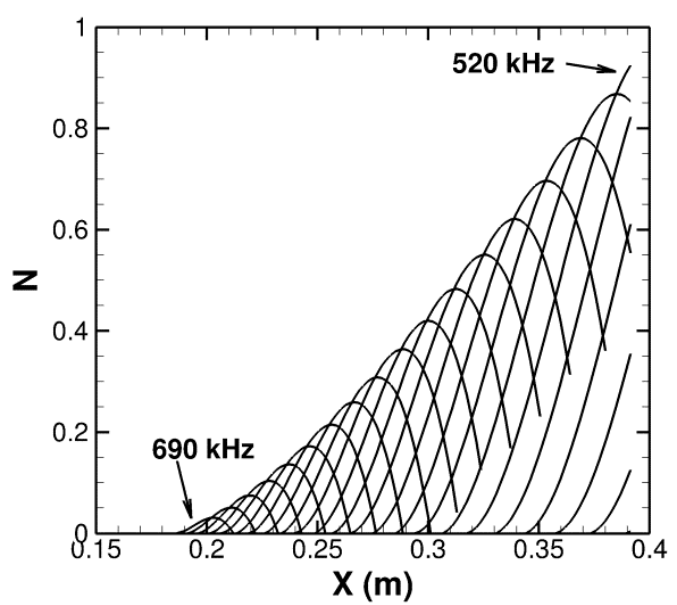

(a) Along major axis meridian.

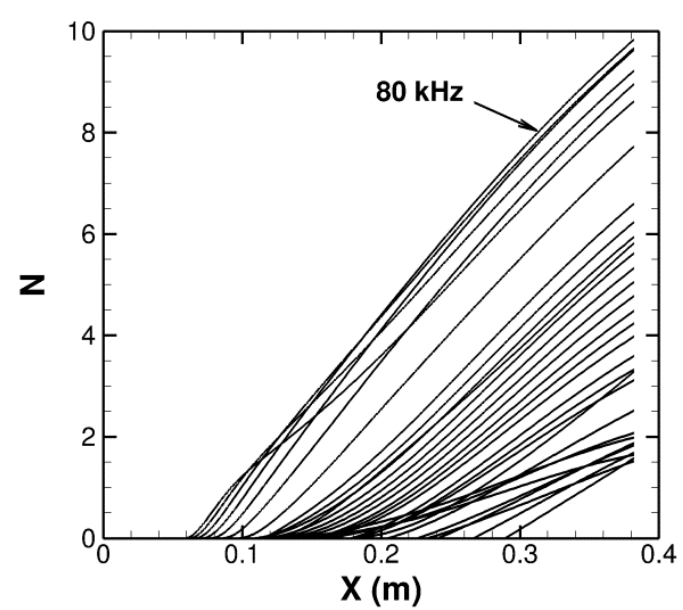

(b) Along minor axis meridian.

Figure 15. N-factor curves along the major and minor axis meridians.

The crossflow instability becomes important in characterizing the transition mechanism on the surface of the elliptic cone away from the attachment line. $\mathrm{N}$-factor computations along streamlines show that the maximum $\mathrm{N}$-factor at the end of the cone for stationary crossflow instability is approximately 9 as shown in Fig. 16. Fig. 17 confirms that the longitudinal streaks observed over the aft portion of the cone in the oil flow visualization from Refs 18 and 19 are very closely (albeit not exactly) aligned with the pattern of inviscid streamlines. Unfortunately, neither the oil flow images nor the TSP measurements at the Case 1 Reynolds number allowed quantitative estimates of the amplitudes of observed stationary streaks. A comparison with the measurements at higher Reynolds numbers, where limited quantitative analysis of the stationary modes became feasible, is deferred to the follow-on effort. On the other hand, measurements of unsteady pressure disturbances at the sensor 1 location in Fig. 16 provided useful information regarding the local state of the boundary layer as well as the frequency spectrum of pretransitional disturbances. In particular, the surface pressure spectra indicated a disturbed laminar flow at the sensor 1 location for Case 1. Thus, the likely contribution of various non-stationary instability modes to the sensor 1 signal is examined below.

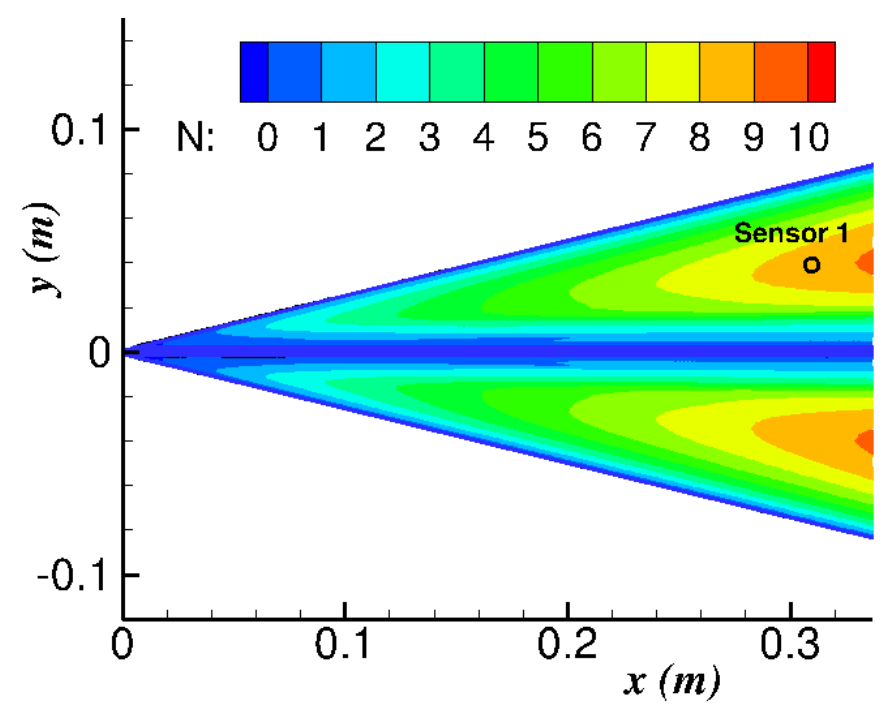

Figure 16. $\mathbf{N}$-factor contours for stationary crossflow mode disturbances. Sensor 1 is located at $(0.3125,0.0371)$ meters. 


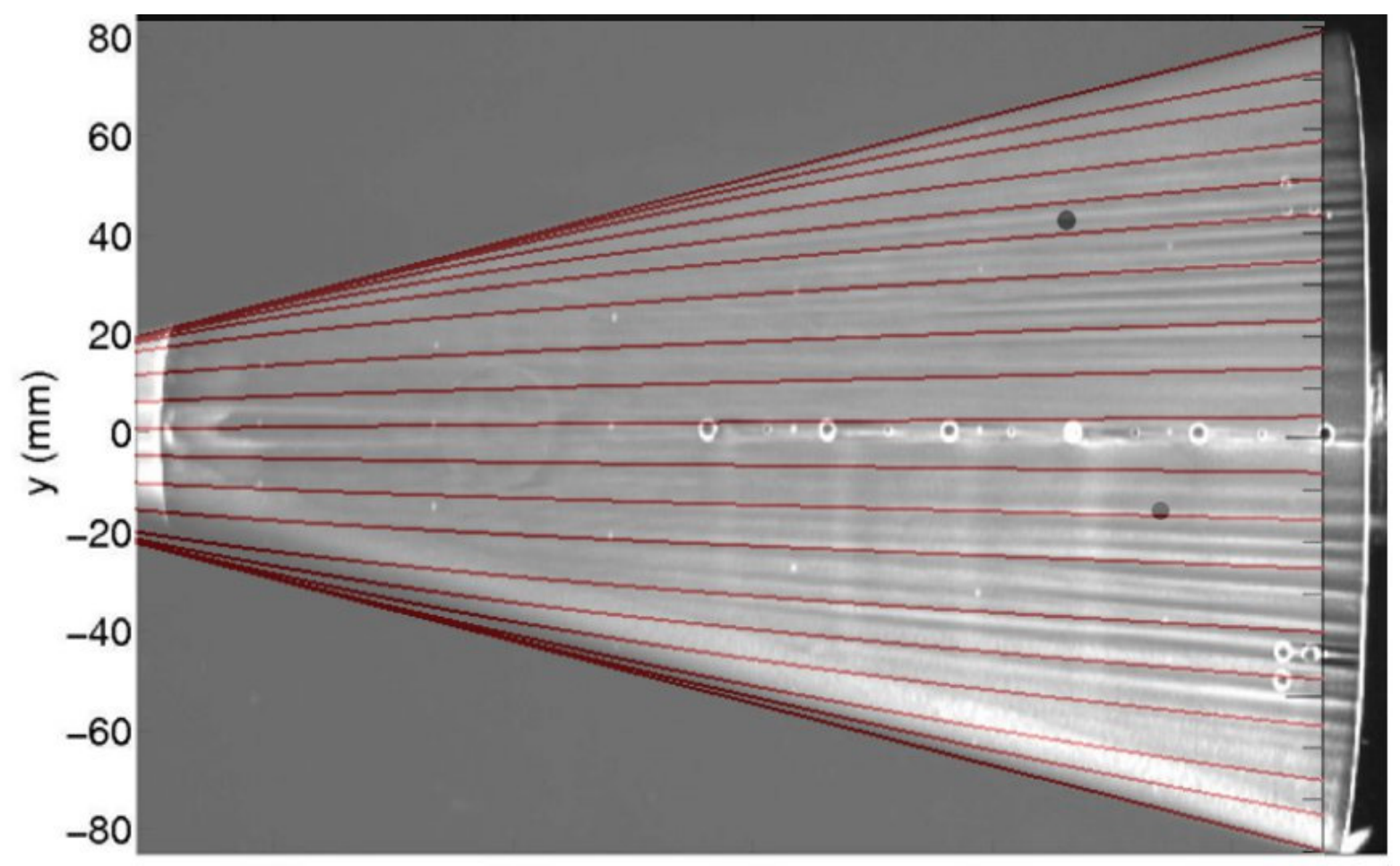

Figure 17. Oil flow visualization from Ref. 18 overlaid with inviscid streamlines used for $\mathbf{N}$-factor calculation (indicated by red lines)

Figure 18 compares the computed and experimentally measured frequency spectra at the sensor 1 location (Fig.16) from wind tunnel measurements of Borg et al. ${ }^{18,19}$ for $\mathrm{Re}=8.1 \times 10^{6} / \mathrm{m}$. The quasi-parallel Nfactor (with both streamwise and transverse curvature accounted for) has been computed by integrating along the streamline that passes through the sensor 1 location. The red and green curves correspond to two different ways of integrating the disturbance growth along the selected trajectory. In the first approach (red curve), the $\mathrm{N}$-factor for each frequency is calculated by integrating the maximized growth rate with respect to spanwise wavenumbers. In the second approach (green curve), the N-factor distribution is computed for various fixed azimuthal wavenumbers based on the local radius of the elliptic cross section. The maximum value of $\mathrm{N}$ factor at the sensor 1 location is selected for each frequency. Larger $\mathrm{N}$ values correspond to larger integrated growth for a particular frequency over the selected streamline. The computed lower frequency lobe corresponds to crossflow instability and the higher frequency but lower peak amplification (the blue, smaller peak over the $\mathrm{N}$-factor lobe) corresponds to the second mode instability. The peak traveling crossflow growth (maximum $\mathrm{N}$ factor) frequency around 40 $\mathrm{kHz}$ is quite close to the peak unsteady frequency from the experimental measurements. The computed spectrum using modal growth appears to agree with the measurement slightly better than the one obtained by integrating the maximized growth rate. The presence of second mode in the higher frequency range is also evident. These second mode waves, nevertheless, have a lower overall growth and only begin to amplify much further downstream from the leading edge. These relatively high frequency waves may play a role in inducing high-frequency secondary instability of highly amplified stationary crossflow instability waves near the end of the model. 


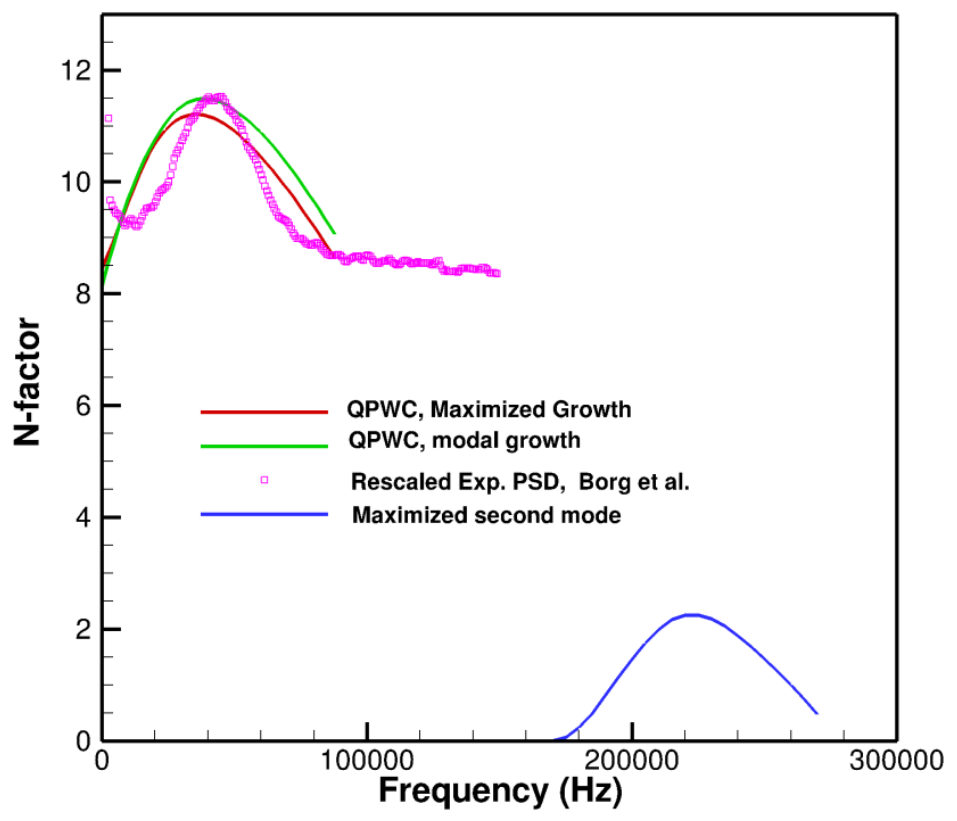

Figure 19. Comparison of computed and experimental frequency spectra at sensor 1 location from wind tunnel experiment of Borg et al. ${ }^{18}$ for $\mathrm{Re}=8.1 \times 10^{6} / \mathrm{m}$. Wind tunnel PSD data has been rescale to fit the computed $\mathrm{N}$-factor range.

The mode shapes of pressure and streamwise velocity fluctuations associated with the most amplified crossflow disturbance ( $\mathrm{f} \approx 40 \mathrm{kHz}$ ) at the sensor location are shown in Fig. 20. These mode shapes are normalized so that the peak amplitude of temperature fluctuations is equal to unity. According to Fig. 20, a non-dimensional surface pressure fluctuation of 0.0008 corresponds to a non-dimensional peak streamwise velocity fluctuation of approximately 0.127 . The broadband peak in the measured surface pressure spectrum from Ref. 18 is estimated to correspond to an r.m.s. pressure fluctuation of approximately 4.6 Pa. If, for simplicity, this energy were to be lumped into the single disturbance mode plotted in Fig. 20, then the corresponding value of r.m.s. velocity amplitude would be approximately 14 $\mathrm{m} / \mathrm{s}$, i.e., 1.6 percent of the free-stream speed. Analysis of the measured data by Borg et al. ${ }^{18}$ suggested an imminent onset of transition behind the sensor 1 location. However, the estimated amplitude of traveling crossflow perturbations appears insufficient to cause transition onset via traveling crossflow as the primary instability mechanism ${ }^{25}$. The fact that no additional peaks corresponding to the harmonics of the main peak near $40 \mathrm{kHz}$ were present in the sensor 1 spectrum (Fig. 19) further supports this inference. Therefore, the likely cause for transition onset under quiet flow conditions at the Case 1 Reynolds number is the stationary crossflow modes. (In this context, it is interesting to observe that the sensor 1 location is very close to the locus of most amplified stationary disturbances from Fig. 16.) The frequency bandwidth of the surface pressure transducer is below the estimated frequency range of the high-frequency secondary instabilities of the stationary vortex modes and, therefore, the available data cannot provide additional clues concerning whether the inferred onset of transition behind the sensor 1 location was caused by the secondary instabilities of stationary modes or by an interaction between the stationary and non-stationary crossflow modes. 


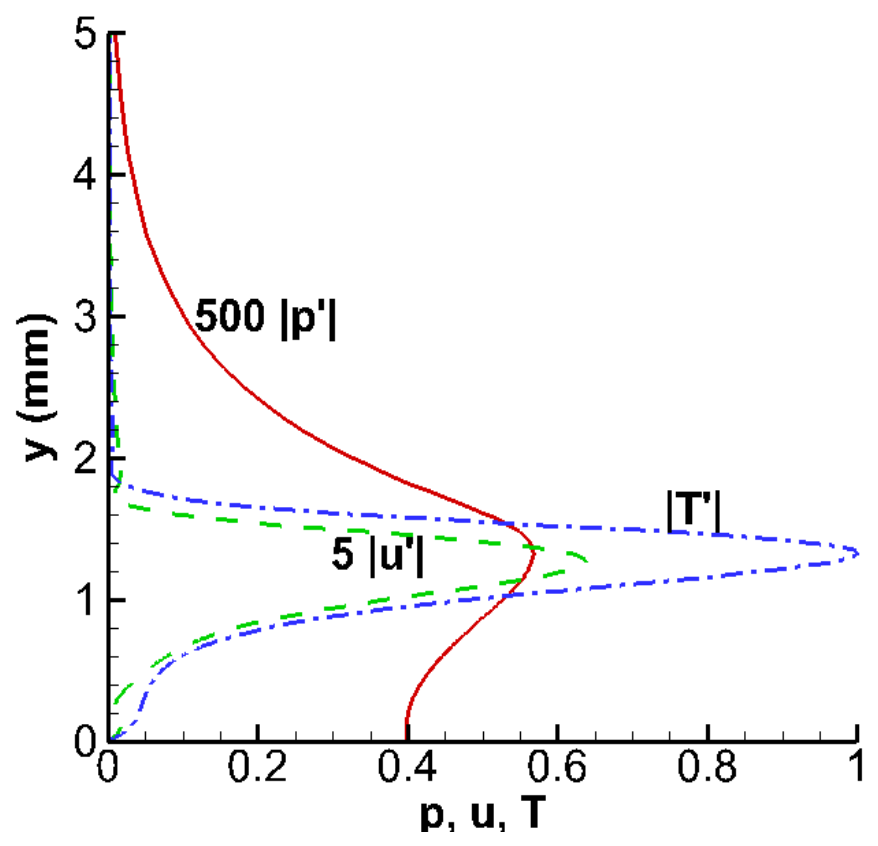

Figure 20. Suitably non-dimensionalized wall-normal mode shapes of traveling crossflow instability at the most amplified frequency $(f=40 \mathrm{kHz})$ at Sensor 1 location.

\section{Conclusions}

The HIFiRE-1 flight experiment by AFRL has provided a valuable and, in many ways, a unique database for boundary layer transition over a circular cone model at varying Mach number, Reynolds number, and angle of attack. Based on measurements over a relevant subset of the ascent trajectory, the onset of transition due to second mode instabilities is found to correlate with an N-factor of approximately 13.5. Transition at early times and lower Mach numbers is believed to be influenced by step excrescences on the model. Due to the higher angle of attack during the descending phase, instability characteristics along the leeward line of symmetry are altered substantially and strong crossflow instability exists in between the windward and leeward planes of symmetry. Linear stability correlations for strongly inhomogeneous 3D boundary layers involve ad hoc assumptions that must be substantiated via case by case comparisons with numerical simulations and/or experimental measurements. Preliminary findings presented herein confirm the presence of a moderate instability at $t=481.3$ seconds. The locations of peak $\mathrm{N}$-factor values and corresponding azimuthal wavenumbers are approximately consistent with the findings based on the analysis of the flight measurements. This finding lends credence to the belief that the higher amplitude, quasi-periodic fluctuations measured by the high frequency transducers are likely to be associated with stationary crossflow instability.

The substantial uncertainty in angle of attack during the reentry phase limits the extent of comparison between flight data and computational predictions. Future computations will address the sensitivity of the computed results to the angle of attack as well as direct numerical simulations pertaining to the effect of crossflow separation on the propagation of both crossflow and second mode instabilities.

Extensive grid convergence analysis was used to demonstrate the existence of multiple, streamwise elongated (i.e., streak-like) vortex structures in the vicinity of the minor axis plane of symmetry as an intrinsic feature of the laminar basic state over the HIFiRE-5 configuration (and, by analogy, other flows involving crossflow driven secondary flow interacting with a symmetry plane). For the HIFiRE-5 wind tunnel model at 38.1 percent scale, the peak $\mathrm{N}$-factor for stationary crossflow instability reaches a value 
of 9 under suitable yet ad hoc assumptions regarding the closure of the eigenvalue problem for the stability of a 3D flow and/or the choice of integration trajectories for the $\mathrm{N}$-factor calculation. The maximum $\mathrm{N}$-factor for the traveling crossflow instability is over 11 and the peak frequency predicted in this manner compares well with measurements by sensor 1. DNS computations will be performed in follow-on effort to provide additional insights into the evolution of instabilities over the model surface and, in particular, into the effects of the streak-like structures as well as to improve the assumptions underlying practical applications of classical stability analysis.

\section{ACKNOWLEDGMENT}

The work of NASA authors was performed as part of the Aerodynamics, Aerothermodynamics, and Plasma Dynamics (AAP) element of the Hypersonics Project of NASA's Fundamental Aeronautics Program (FAP).

\section{REFERENCES}

1 Kimmel, R. L., Adamczak, D., Gaitonde, D., Rougeux, A., and Haynes, J. R., "HIFiRE-1 Boundary Layer Transition Experiment Design," AIAA Paper 2007-534, 2007.

2 Kimmel, R. L., “Aerothermal Design for HIFiRE-1 Flight Vehicle,” AIAA Paper 2008-4034, 2008.

3 Kimmel, R. L., Adamczak, D., and DSTO AVD Brisbane Team, "HIFiRE Preliminary Aerothermodynamic Measurements," AIAA Paper 2011-3413, 2011.

4 Adamczak, D., Kimmel, R. L., and DSTO AVD Brisbane Team, "HIFiRE-1 Trajectory Estimation and Preliminary Experimental Results," AIAA paper 2011-2358, 2011.

5 Stanfield, S., Kimmel, R. L., and Adamczak, D., "HIFiRE-1 Flight Data Analysis: Boundary Layer Transition Experiment During Reentry," AIAA Paper 2012-1087, 2012.

6 Li, F., Choudhari, M., Chang, C.-L., Kimmel, R., Adamczak, D., and Smith, M., "Transition Analysis for the HIFiRE-1 Flight Experiment," AIAA Paper 2011-3414, 2011.

7 Kimmel, R., Adamczak, D., Berger, K. and Choudhari, M. "HIFiRE-5 Flight Vehicle Design," AIAA Paper 2010-4985.

8 Choudhari, M., Chang, C.-L., Jentink, T., Li, F., Berger, K., Candler, G., and Kimmel, R., "Transition Analysis for the HIFiRE-5 Vehicle," AIAA Paper 2009-4056, 2009.

9 Litton, D.K., Edwards, J.R. and White, J.A., "Algorithmic Enhancements to the VULCAN Navier-Stokes Solver," AIAA 2003-3979, June, 2003. (Also: http://vulcan-cfd.larc.nasa.gov (Aug. 18, 2011))

10 Chang, C.-L., "LASTRAC.3d: Transition Prediction in 3D Boundary Layers," AIAA Paper 2004-2542, 2004.

11 Wheaton, B. M., Juliano, T. J., Berridge, D. C., Chou, A., Gilbert, P. L., Casper, K. M., Sheen, L. E. and Schneider, S. P., "Instability and Transition Measurements in the Mach-6 Quiet Tunnel," AIAA Paper 2009-3559, 2009.

12 Li, F., Choudhari, M., Chang, C.-L. and White, J., "Analysis of Instabilities in NonAxisymmetric Hypersonic Boundary Layers over Cones," AIAA Paper 2010-4643, 2010.

13 Malik, M., Li, F. and Choudhari, M., "Analysis of Crossflow Transition Flight Experiment aboard Pegasus Launch Vehicle," AIAA Paper 2007-4487, 2007.

14 Tracy, R.R., "Hypersonic Flow Over a Yawed Circular Cone," California Institute of Technology, Aeronautical Lab., Memo 69, 1963.

15 Lin, T.C. and Rubin, S.G., "Viscous Flow over a Cone at Moderate Incidence. II: Supersonic Boundary Layer," J. Fluid Mech., Vol. 59, March 1973, pp. 593-620.

16 Choudhari, M., Li, F., Wu, M., Chang, C.-L. and Edwards, J.A., "Laminar Turbulent Transition behind Discrete Roughness Elements in a High-Speed Boundary-Layer," AIAA Paper 2010-1575, 2010. 
Carpenter, A.L., Saric, W.S. and Reed, H.L., "Laminar Flow Control on a Swept Wing with Distributed Roughness," AIAA Paper 2008-7335, 2008.

18 Borg, Matthew P., Kimmel, Roger and Stanfield, Scott, "Attachment-Line and Crossflow Instability in a Quite Hypersonic Wind Tunnel,” AIAA Paper 2011-3247, 2011.

19 Borg, Matthew P., Kimmel, Roger and Stanfield, Scott, "Stationary Crossflow Instability and Co-Located Traveling Disturbances on an Elliptic Cone in Quite Hypersonic Flow," Paper 34, ATV-200 Specialist Meeting on Hypersonic Transition, San Diego, CA, April 2012.

20 Tokugawa, N., Choudhari, M., Ishikawa, H., Ueda, Y., Fujii, K., Atobe, T., Li, F., Chang, C.L. and White, J., "Transition Along Leeward Ray of Axisymmetric Bodies at Incidence in Supersonic Flow," To be presented at the AIAA Fluid Dynamics Conference, New Orleans, 2012.

21 Choudhari, M., Tokugawa, N., Li, F., Chang, C.-L., White, J., Ishikawa, H., Ueda, Y. Atobe, T. and Fujii, K., "Computational Investigation of Supersonic Boundary Layer Transition over Canonical Fuselage Nose Configurations," To be presented at the $7^{\text {th }}$ International Conference on Computational Fluid Dynamics, Island of Hawaii, June 2012.

22 Kegerise, M., King, R., Owens, L. Choudhari, M. Norris, A., Li. F. and Chang, C.-L. "Wake Instability Behind a Discrete Roughness Element in High Speed Boundary Layers," Paper 28, ATV-200 Specialist Meeting on Hypersonic Transition, San Diego, CA, April 2012.

23 Li, F. and Choudhari, M., "Spatially Developing Secondary Instabilities and Attachment Line Instability in Supersonic Boundary Layers," AIAA Paper 2008-590, 2008.

24 Li, F. and Choudhari, M., "Spatially Developing Secondary Instabilities in Compressible Swept Airfoil Boundary Layers," Theo. Comput. Fluid Dyn., Vol. 25, pp. 65-84, June, 2011.

25 Malik, M., Li, F. and Chang, C.-L., "Crossflow Disturbances in Three Dimensional Boundary Layers: Nonlinear Development, Wave Interaction and Secondary Instability," J. Fluid Mech., Vol. 268, p. 1, 1994. 